T. OTSLK.

KODAI MATH. J.

12 (1989), 346-391

\title{
A CERTAIN PROPERTY OF GEODESICS OF THE FAMILY OF RIEMANNIAN MANIFOLDS $O_{n}^{2}(X)$
}

\author{
Dedicated to Professor Shiing-Shen Chern on his 77th birthday
}

\author{
By Tominosuke OTsUKI
}

§ 0. Introduction. This is a continuation of Part (IX) ([22]) with the same title by the present author which proved the following conjecture is true for $2.4 \leqq n \leqq 4.5$ and exactly the final one of the series $(\mathrm{I})-(\mathrm{X})$. He will show that this conjecture is also true for $2 \leqq n \leqq 2.4$ in the present paper by developing a new method which is applicable for all values of $n \geqq 2.4$. As stated at the end of the previous one, the principle used until now could not hold for $2 \leqq n \leqq 2.38$. We shall also use the same notation in the previous papers (I)-(IX). Any geodesic of the 2-dimensional Riemannian manifolds $O_{n}^{2}$ defined on the unit disk $u^{2}+v^{2}<1$ of the $u v$-plane by the metric:

$$
d s^{2}=\left(1-u^{2}-v^{2}\right)^{n-2}\left\{\left(1-v^{2}\right) d u^{2}+2 u v d u d v+\left(1-u^{2}\right) d v^{2}\right\}
$$

has the support function $x(t)$ which is a solution of the non linear differential equation of order 2([6]):

$$
n x\left(1-x^{2}\right) \frac{d^{2} x}{d t^{2}}+\left(\frac{d x}{d t}\right)^{2}+\left(1-x^{2}\right)\left(n x^{2}-1\right)=0
$$

When the parameter $n>1$, any non-constant solution $x(t)$ of $(E)$ such that $x^{2}+x^{\prime 2}<1$ is periodic and its period $T$ is given by the improper integral ([10]):

$$
T=\sqrt{n c} \int_{x_{0}}^{x_{1}} \frac{d x}{x \sqrt{(n-x)\left\{x(n-x)^{n-1}-c\right\}}},
$$

where $x_{0}=n\{\min x(t)\}^{2}, x_{1}=n\{\max x(t)\}^{2}, 0<x_{0}<1<x_{1}<n$ and $c=x_{0}\left(n-x_{0}\right)^{n-1}$ $=x_{1}\left(n-x_{1}\right)^{n-1}$.

CONJECTURE C. The period $T$ as a function of $\tau=\left(x_{1}-1\right) /(n-1)$ and $n$ is monotone decreasing with respect to $n(>2)$ for any fixed $\tau(0<\tau<1)$.

\section{$\S 1$. Preliminaries.}

Setting $T=\Omega(\tau, n)$, we have the formulas

Received November 11, 1988. 
(1.1) $\quad \frac{\partial \Omega(\tau, n)}{\partial n}=-\frac{\sqrt{c}}{2(B-c) n \sqrt{n}} \int_{x_{0}}^{1} \frac{(1-x) \sqrt{x(n-x)^{n-1}-c}}{x^{2}(n-x)^{n}} V\left(x, x_{1}\right) d x$

((7.4) and Proposition 3 in (III) and (1.12), (1.16) in (IX)), where $B=(n-1)^{n-1}$ and

$$
\begin{gathered}
V\left(x, x_{1}\right)=\frac{x^{2} \sqrt{n-x} F_{2}(x)}{(1-x)^{5}}\left\{\tilde{\lambda}(x)-\tilde{\lambda}\left(x_{1}\right)\right\} \\
+\frac{X^{2} \sqrt{n-X F_{2}}(X)}{(X-1)^{5}}\left\{\tilde{\lambda}(X)-\tilde{\lambda}\left(x_{1}\right)\right\}-\eta(x)+\eta(X), \\
F_{2}(z)=-B P_{2}(z)+(n-z)^{n-1} P_{3}(z), \\
P_{2}(z)=(2 n+1) z^{2}-2\left(2 n^{2}+5 n-4\right) z+(4 n-1)(4 n-3), \\
P_{3}(z)=-(n-1) z^{3}+\left(2 n^{2}-7 n+8\right) z^{2}+(n-3)(4 n-1) z+3 n(2 n-1), \\
\eta(z)=\frac{z \sqrt{n-z}}{(n-1)(z-1)^{3}}\left\{-B \tilde{Q}_{1}(z)+(n-z)^{n-1} \tilde{Q}_{2}(z)\right\}, \\
\tilde{Q}_{1}(z)=-\left(4 n^{2}+2 n-3\right) z+(4 n-1)(4 n-3), \\
\tilde{Q}_{2}(z)=(n-1)(2 n-3) z^{2}+\left(4 n^{2}-10 n+3\right) z+3 n(2 n-1), \\
\tilde{\lambda}(z)=\log (n-z)+\frac{n z-1}{(n-1) z}, \quad \phi(z)=z(n-z)^{n-1},
\end{gathered}
$$

and $X=X_{n}(x), 0<x<1<X<n$, defined by $\phi(x)=\phi(X)$.

By Lemma 8.1 in (III), we know the following:

FACT 1. For $0<x<1, V\left(x, x_{1}\right)$ is increasing with respect to $x_{1}$ in $X_{n}(x) \leqq$ $x_{1}<n$;

FACT 2. We have

$$
\frac{1}{B} V\left(x, X_{n}(x)\right)=\frac{n\left(4 n^{2}-10 n+3\right)}{(n-1)^{3}} \sqrt{n-X_{n}(x)}\left\{1+O\left(n-X_{n}(x)\right)\right\}
$$

near $x=0$, which implies $V\left(x, X_{n}(x)\right)$ is negative near $x=0$, when $2<n<$ $(5+\sqrt{13}) / 4=2.151387819 \cdots((8.10)$ in $I I I))$;

FACT 3. We have

$$
\frac{1}{B} V\left(x, X_{n}(x)\right)=\frac{n(2 n-1)\left(n^{2}-n-3\right)}{6(n-1)^{2} \sqrt{n-1}}(1-x)\{1+O(1-x)\}
$$

near $x=1$, which implies $V\left(x, X_{n}(x)\right)$ is negative near $x=1$, when $2<n<$ $(1+\sqrt{13}) / 2=2.302775638 \cdots((8.22)$ in $(I I I))$.

We have shown that Conjecture $C$ is true for $2.4 \leqq n<\infty$ by proving that 
$V\left(x, X_{n}(x)\right)>0$ for $0<x<1$ by Fact 1 in (I)-(IX). Now, we show that it is still alive for $2 \leqq n<2.4$ by the following

PROPOSITION 1.

$$
\left.\frac{\partial \Omega(\tau, n)}{\partial n}\right|_{n=2}<0 \quad \text { for } 0<x<1
$$

Proof. By (1.3)-(1.9) we have $B=1$ and

$$
\begin{gathered}
P_{2}(z)=5 z^{2}-28 z+35, \quad P_{3}(z)=-z^{3}+2 z^{2}-7 z+18, \\
F_{2}(z)=-P_{2}(z)+(2-z) P_{3}(z)=(z-1)^{4}, \\
\tilde{Q}_{1}(z)=-17 z+35, \quad \tilde{Q}_{2}(z)=z^{2}-z+18, \\
-\tilde{Q}_{1}(z)+(2-z) \tilde{Q}_{2}(z)=-(z-1)^{3}, \quad \eta(z)=-z \sqrt{2-z}, \\
\tilde{\lambda}(z)-\tilde{\lambda}\left(x_{1}\right)=\log \frac{2-z}{2-x_{1}}-\frac{1}{z}+\frac{1}{x_{1}}
\end{gathered}
$$

and

$$
\begin{aligned}
V\left(x, x_{1}\right)= & \frac{x^{2} \sqrt{2-x}}{1-x}\left\{\log \frac{2-x}{2-x_{1}}-\frac{1}{x}+\frac{1}{x_{1}}\right\} \\
& +\frac{X^{2} \sqrt{2-X}}{X-1}\left\{\log \frac{2-X}{2-x_{1}}-\frac{1}{X}+\frac{1}{x_{1}}\right\}+x \sqrt{2-x}-X \sqrt{2-X} .
\end{aligned}
$$

Since we have $X=2-x, x_{1}=2-x_{0}$, we have

$$
\begin{aligned}
V\left(x, x_{1}\right)= & \frac{x^{2} \sqrt{2-x}}{1-x}\left\{\log \frac{2-x}{x_{0}}-\frac{1}{x}+\frac{1}{2-x_{0}}\right\} \\
& +\frac{(2-x)^{2} \sqrt{x}}{1-x}\left\{\log \frac{x}{x_{0}}-\frac{1}{2-x}+\frac{1}{2-x_{0}}\right\}+x \sqrt{2-x}-(2-x) \sqrt{x} \\
= & \frac{\sqrt{x(2-x)}}{1-x}\left[x^{3 / 2}\left(\log \frac{2-x}{x_{0}}-\frac{1-x_{0}}{2-x_{0}}\right)+(2-x)^{3 / 2}\left(\log \frac{x}{x_{0}}-\frac{1-x_{0}}{2-x_{0}}\right)\right]
\end{aligned}
$$

and hence

$$
\begin{aligned}
& \left.\frac{\partial \Omega(\tau, n)}{\partial n}\right|_{n=2}=-\frac{\sqrt{c}}{4 \sqrt{2(1-c)}} \int_{x_{0}}^{1} \frac{\sqrt{x(2-x)-c}}{(x(2-x))^{3 / 2}} \\
& \times\left[x^{3 / 2}\left(\log \frac{2-x}{x_{0}}-\frac{1-x_{0}}{2-x_{0}}\right)+(2-x)^{3 / 2}\left(\log \frac{x}{x_{0}}-\frac{1-x_{0}}{2-x_{0}}\right)\right] d x \\
& =-\frac{\sqrt{c}}{4 \sqrt{2(1-c)}} \int_{x_{0}}^{2-x_{0}} \frac{\sqrt{x(2-x)-c}}{(x(2-x))^{3 / 2}} x^{3 / 2}\left(\log \frac{2-x}{x_{0}}-\frac{1-x_{0}}{2-x_{0}}\right) d x .
\end{aligned}
$$

Putting $x_{0}=1-a$, and $x=1+t$, we obtain 


$$
\begin{aligned}
I(a) & =\int_{x_{0}}^{2-x_{0}} \frac{\sqrt{x(2-x)-c}}{(x(2-x))^{3 / 2}} x^{3 / 2}\left(\log \frac{2-x}{x_{0}}-\frac{1-x_{0}}{2-x_{0}}\right) d x \\
& =\int_{-a}^{a} \frac{\sqrt{a^{2}-t^{2}}}{(1-t)^{3 / 2}}\left(\log \frac{1-t}{1-a}-\frac{a}{1+a}\right) d t
\end{aligned}
$$

where $0<a<1$. It is sufficient to prove $I(a)>0$.

Since we have

$$
\left\{\frac{2}{\sqrt{1-t}}\left(\log \frac{1-t}{1-a}+\frac{2+a}{1+a}\right)\right\}^{\prime}=\frac{1}{(1-t)^{3 / 2}}\left(\log \frac{1-t}{1-a}-\frac{a}{1+a}\right),
$$

we obtain from (1.13)

$$
\begin{aligned}
I(a)= & 2 \int_{-a}^{a} \frac{t}{\sqrt{(1-t)\left(a^{2}-t^{2}\right)}}\left(\log \frac{1-t}{1-a}+\frac{2+a}{1+a}\right) d t \\
= & 2 \int_{0}^{a} \frac{t}{\sqrt{a^{2}-t^{2}}}\left\{\frac{1}{\sqrt{1-t}}\left(\log \frac{1-t}{1-a}+\frac{2+a}{1+a}\right)\right. \\
& \left.-\frac{1}{\sqrt{1+t}}\left(\log \frac{1+t}{1-a}+\frac{2+a}{1+a}\right)\right\} d t
\end{aligned}
$$

by the integration by parts.

Setting for a fixed $a(0<a<1)$

$$
f(t):=\frac{1}{1-t}\left(\log \frac{1-t}{1-a}+\frac{2+a}{1+a}\right)^{2} \quad \text { for }-a \leqq t \leqq a,
$$

we prove that

$$
f(t)>f(-t) \quad \text { for small } t>0
$$

Since we have

$$
f^{\prime}(t)=\frac{1}{(1-t)^{2}}\left(\log \frac{1-t}{1-a}+\frac{2+a}{1+a}\right)\left(\log \frac{1-t}{1-a}-\frac{a}{1+a}\right)
$$

and

$$
f^{\prime}(0)=\left(\log \frac{1}{1-a}+\frac{2+a}{1+a}\right)\left(\log \frac{1}{1-a}-\frac{a}{1+a}\right)>0
$$

which implies (1.16).

Next we prove that

$$
f(a)>f(-a)
$$

Since we have

$$
f(a)=\frac{1}{1-a}\left(\frac{2+a}{1+a}\right)^{2} \text { and } \quad f(-a)=\frac{1}{1+a}\left(\log \frac{1+a}{1-a}+\frac{2+a}{1+a}\right)^{2}
$$


and so $f(a)>f(-a)$ is equivalent to

$$
\frac{2+a}{1+a}\left(\sqrt{\frac{1+a}{1-a}}-1\right)>\log \frac{1+a}{1-a} \quad \text { for } 0<a<1 \text {. }
$$

Setting $b=(1+a) /(1-a)$ and

$$
g(b):=\frac{2+a}{1+a}\left(\sqrt{\frac{1+a}{1-a}}-1\right)-\log \frac{1+a}{1-a}=\frac{3 b+1}{2 b}(\sqrt{b}-1)-\log b,
$$

we have $b>1$ for $0<a<1$ and

$$
\begin{gathered}
g(1)=0, \\
g^{\prime}(b)=\frac{1}{4 b^{2}}(\sqrt{b}-1)^{2}(3 \sqrt{b}+2)>0 \quad \text { for } b>1,
\end{gathered}
$$

which implies (1.17).

Last, we prove

$$
f(t)>f(-t) \quad \text { for } 0<t<a .
$$

If this inequality does not hold, there exist $0<t_{1} \leqq t_{2}<a$ such that

and

$$
f\left(t_{1}\right)=f\left(-t_{1}\right) \text { and } f^{\prime}\left(t_{1}\right)+f^{\prime}\left(-t_{1}\right) \leqq 0
$$

$$
f\left(t_{2}\right)=f\left(-t_{2}\right) \text { and } f^{\prime}\left(t_{2}\right)+f^{\prime}\left(-t_{2}\right) \geqq 0 .
$$

Since we obtain easily from (1.15)

$$
\begin{aligned}
f^{\prime}(t)+f^{\prime}(-t)= & \frac{1}{(1-t)^{2}}\left(\log \frac{1-t}{1-a}\right)^{2}-\frac{2}{(1-t)^{2}}\left(\log \frac{1-t}{1-a}+\frac{2+a}{1+a}\right) \\
& +\frac{1}{(1+t)^{2}}\left(\log \frac{1+t}{1-a}+\frac{2+a}{1+a}\right)^{2}-\frac{2}{(1+t)^{2}}\left(\log \frac{1+t}{1-a}+\frac{2+a}{1+a}\right)
\end{aligned}
$$

which can be written at $t=t_{1}$ and $t_{2}$ as

$$
=\frac{2 h(t)}{(1-t)^{2}(1+t)}\left(\log \frac{1-t}{1-a}+\frac{2+a}{1+a}\right) \text {. }
$$

where

$$
h(t):=\log \frac{1-t}{1-a}+\frac{2+a}{1+a}-\frac{(1+t)^{3 / 2}+(1-t)^{3 / 2}}{\sqrt{ } 1+t} .
$$

Regarding $h(t)$, we have

$$
h(0)=\log \frac{1}{1-a}-\frac{a}{1+a}>0
$$

and

$$
h^{\prime}(t)=-\frac{2-t}{1-t}+\frac{3}{2} \sqrt{\frac{1-t}{1+t}}+\frac{1}{2}\left(\frac{1-t}{1+t}\right)^{3 / 2},
$$


which can be written as

$$
\begin{gathered}
-\frac{1+3 u}{2 u}+\frac{3}{2} u^{1 / 2}+\frac{1}{2} u^{3 / 2} \\
=\frac{1}{2 u}\left(u^{1 / 2}-1\right)\left(u^{2}+u^{3 / 2}+4 u+u^{1 / 2}+1\right),
\end{gathered}
$$

where $u=(1-t) /(1+t)$. Since $0<u \leqq 1$ for $0 \leqq t<1$, we have $h^{\prime}(t)<0$ for $0<t<1$. Hence $h(t)$ is decreasing in $0<t<a$ and

$$
h(a)=\frac{2+a}{1+a}-\frac{(1+a)^{3 / 2}+(1-a)^{3 / 2}}{\sqrt{1+a}}:=G(a) .
$$

Regarding $G(a)$, we have

and

$$
G(0)=0
$$

$$
\begin{aligned}
G^{\prime}(a) & =-\frac{1}{(1+a)^{2}}-1+\frac{3}{2} \sqrt{\frac{1-a}{1+a}}+\frac{1}{2}\left(\frac{1-a}{1+a}\right)^{3 / 2} \\
& =-\frac{1}{4}\left(1-b^{1 / 2}\right)\left(5-b^{1 / 2}+b-b^{3 / 2}\right),
\end{aligned}
$$

where $b=(1-a) /(1+a)$ and $0<b<1$ for $0<a<1$. Hence we obtain

$$
G^{\prime}(a)<0 \text { and } G(a)<0 \text { for } 0<a<1 .
$$

Thus we see that there exists a unique $t=\gamma(a)$ such that

$$
\begin{array}{ll}
h(t)>0 & \text { for } 0 \leqq t<\gamma(a) \\
h(t)<0 & \text { for } \gamma(a)<t<a .
\end{array}
$$

Since we have $h\left(t_{1}\right) \leqq 0$ and $h\left(t_{2}\right) \geqq 0$, it must be

and hence there

$$
t_{1}=t_{2}=\gamma(a),
$$

$$
f(t)=f(-t) \text { and } f^{\prime}(t)+f^{\prime}(-t)=0,
$$

from which we obtain

$$
\log \frac{1+t}{1-t}=\left\{(1+t)^{3 / 2}+(1-t)^{3 / 2}\right\}\left\{\frac{1}{\sqrt{1-t}}-\frac{1}{\sqrt{1+t}}\right\}
$$

at $t=t_{1}=t_{2}$. Using $v=(1+t) /(1-t)=x^{2}$, we have

$$
\begin{gathered}
\left\{(1+t)^{3 / 2}+(1-t)^{3 / 2}\right\}\left\{\frac{1}{\sqrt{ } 1-t}-\frac{1}{\sqrt{ } 1+t}\right\}-\log \frac{1+t}{1-t} \\
=\frac{2}{v+1}\left(v^{3 / 2}+1\right)\left(1-\frac{1}{v^{1 / 2}}\right)-\log v
\end{gathered}
$$




$$
=2\left[\frac{(x-1)\left(x^{3}+1\right)}{x\left(x^{2}+1\right)}-\log x\right] \text {. }
$$

Since we have easily

$$
\left(\frac{(x-1)\left(x^{3}+1\right)}{x\left(x^{2}+1\right)}-\log x\right)^{\prime}=\frac{(x-1)^{2}}{x^{2}\left(x^{2}+1\right)^{2}}\left(x^{4}+x^{3}+4 x^{2}+x+1\right)
$$

and $1<x<\infty$ for $0<t<1$, it must be

$$
\left\{(1+t)^{3 / 2}+(1-t)^{3 / 2}\right\}\left\{\frac{1}{\sqrt{1-t}}-\frac{1}{\sqrt{1+t}}\right\}>\log \frac{1+t}{1-t} \quad \text { for } 0<t<1,
$$

which implies a contradiction for $t_{1}$ and $t_{2}$. Thus we obtain (1.18), which implies $I(a)>0$.

Q.E.D.

In the following, we shall use the methods appeared in the proof of Proposition 1.

§2. The fundamental principle. By (7.10) in (III), we have

$$
V\left(x, x_{1}\right)=\frac{x^{2} N\left(x, x_{1}\right)}{(1-x)^{5} \sqrt{n-x}}+\frac{X^{2} N\left(X, x_{1}\right)}{(X-1)^{5} \sqrt{n-X}},
$$

where

$$
\begin{gathered}
\begin{aligned}
& N\left(z, x_{1}\right):=(n-z) F_{2}(z)\left\{\lambda(z)-\tilde{\lambda}\left(x_{1}\right)\right\}+3(z-1)^{2} f_{0}(z) \\
&- 2 n(z-1)^{3}\{B-\phi(z)\}, \\
& f_{0}(z):=(2 n-1-z) B-(n-z)^{n-1}\left\{n-z+(n-1) z^{2}\right\}, \\
& \lambda(z):=\log (n-z)+\frac{n-1}{n-z}
\end{aligned}
\end{gathered}
$$

and $X=X_{n}(x)$ for $0 \leqq x \leqq 1$. Since we have

$$
\phi^{\prime}(x)=n(1-x)(n-x)^{n-2} \text { and } \frac{1-x}{x(n-x)} d x=-\frac{X-1}{X(n-X)} d X,
$$

we obtain

$$
\begin{aligned}
I & =\int_{x_{0}}^{1} \frac{(1-x) \sqrt{\psi(x)-c}}{x^{2}(n-x)^{n}} V\left(x, x_{1}\right) d x \\
& =\int_{x_{0}}^{x_{1}} \frac{\sqrt{\psi(x)-c} N\left(x, x_{1}\right)}{(x-1)^{4}(n-x)^{n+1 / 2}} d x .
\end{aligned}
$$

Regarding $N\left(x, x_{1}\right)$, we know that $F_{2}(z), f_{0}(z)$ and $B-\phi(z)$ are of order 4,3 and 2 in $z-1$ at $z=1$ by (8.14) and (8.17) in (III) and Lemma 4.1 in (I), respectively, and hence the integrand of the right hand side of (2.5) is real analytic 
at $x=1$. Therefore we set

$$
M\left(x, x_{1}\right):=\int_{1}^{x} \frac{N\left(t, x_{1}\right) d t}{(t-1)^{4}(n-t)^{n+1 / 2}}
$$

and hence by the integration by parts we have

$$
\begin{aligned}
I & =\left[\sqrt{\phi(x)-c} M\left(x, x_{1}\right)\right]_{x_{0}}^{x_{1}}-\frac{n}{2} \int_{x_{0}}^{x_{1}} \frac{(1-x)(n-x)^{n-2}}{\sqrt{\phi(x)-c}} M\left(x, x_{1}\right) d x \\
& =-\frac{n}{2} \int_{x_{0}}^{x_{1}} \frac{(1-x)(n-x)^{n-2}}{\sqrt{\phi(x)-c}} M\left(x, x_{1}\right) d x .
\end{aligned}
$$

Since we have

$$
\begin{aligned}
& \int_{1}^{x_{1}} \frac{(1-x)(n-x)^{n-2}}{\sqrt{\phi(x)-c}} M\left(x, x_{1}\right) d x \\
& \quad=\int_{1}^{x_{1}} \frac{(1-X)(n-X)^{n-2}}{\sqrt{\phi(X)-c}} M\left(X, x_{1}\right) d X \\
& \quad=-\int_{1}^{x_{0}} \frac{(1-X)(n-X)^{n-2}}{\sqrt{\psi(x)-c}} M\left(X, x_{1}\right) \cdot \frac{X(n-X)}{X-1} \cdot \frac{1-x}{x(n-x)} d x \\
& \quad=-\int_{x_{0}}^{1} \frac{(1-x)(n-x)^{n-2}}{\sqrt{\phi(x)-c}} M\left(X, x_{1}\right) d x,
\end{aligned}
$$

we obtain

$$
I=\frac{n}{2} \int_{x_{0}}^{1} \frac{(1-x)(n-x)^{n-2}}{\sqrt{\psi(x)-c}}\left\{M\left(X, x_{1}\right)-M\left(x, x_{1}\right)\right\} d x .
$$

From this formula, we see that if we can prove

$$
M\left(X_{n}(x), x_{1}\right)-M\left(x, x_{1}\right)>0 \text { for } x_{0} \leqq x<1,
$$

then we obtain $I>0$. When $n=2$, we can easily check that

$$
\begin{aligned}
M\left(X, x_{1}\right)-M\left(x, x_{1}\right)= & \frac{2}{\sqrt{1-t}}\left(\log \frac{1-t}{1-a}+\frac{2+a}{1+a}\right) \\
& -\frac{2}{\sqrt{1+t}}\left(\log \frac{1+t}{1-a}+\frac{2+a}{1+a}\right),
\end{aligned}
$$

where $a=1-x_{0}, t=1-x$, and so (\#) corresponds to (1.18).

Now, we set

$$
\rho\left(x, x_{1}\right):=M\left(X_{n}(x), x_{1}\right)-M\left(x, x_{1}\right)=\int_{x}^{x} \frac{N\left(t, x_{1}\right) d t}{(t-1)^{4}(n-t)^{n+1 / 2}}
$$

where $X=X_{n}(x)$.

LEMMA 2.1. i) $\rho\left(1, x_{1}\right)=0$, 
ii) $\rho\left(x, x_{1}\right)>0$ and $\searrow$ for $x<1$ near $x=1$.

Proof. i) is evident. By (8.14), (8.17) in (III), near $t=1$ we have

$$
\begin{aligned}
N\left(t, x_{1}\right)= & (n-t)\left\{\lambda(t)-\tilde{\lambda}\left(x_{1}\right)\right\} \\
& \times\left\{\frac{n\left(n^{2}-n+1\right) B}{6(n-1)}+O(1-t)\right\}(1-t)^{4} \\
& -\left\{\frac{n(2 n-1) B}{2}+O(1-t)\right\}(1-t)^{5} \\
& +2 n\left\{\frac{n(n-1)^{n-2}}{2}+O(1-t)\right\}(1-t)^{5}
\end{aligned}
$$

and hence

$$
\begin{gathered}
\frac{N\left(t, x_{1}\right)}{(t-1)^{4}(n-t)^{n+1 / 2}}=\frac{n\left(n^{2}-n+1\right) B}{6(n-1)}\left\{\lambda(t)-\tilde{\lambda}\left(x_{1}\right)\right\} \frac{1}{(n-t)^{n-1 / 2}}+O(1-t) \\
=\frac{n\left(n^{2}-n+1\right)}{6(n-1)^{3 / 2}}\left\{\lambda(1)-\tilde{\lambda}\left(x_{1}\right)\right\}+O(1-t) .
\end{gathered}
$$

For sufficiently small $\varepsilon>0$, we obtain

$$
\begin{aligned}
\rho\left(1-\varepsilon, x_{1}\right)= & \frac{n\left(n^{2}-n+1\right)}{6(n-1)^{3 / 2}}\left\{\lambda(1)-\tilde{\lambda}\left(x_{1}\right)\right\}\{X(1-\varepsilon)-(1-\varepsilon)\} \\
& +\left[\frac{(1-t)^{2}}{2}\right]_{1-\varepsilon}^{X(1-\varepsilon)} \times O(1) .
\end{aligned}
$$

Since we have

$$
X(1-\varepsilon)=1+\varepsilon+\frac{2(n-2)}{3(n-1)} \varepsilon^{2}+\cdots
$$

by (8.12) in (III),

$$
\begin{aligned}
\rho\left(1-\varepsilon, x_{1}\right) & =\frac{n\left(n^{2}-n+1\right)}{6(n-1)^{3 / 2}}\left\{\lambda(1)-\tilde{\lambda}\left(x_{1}\right)\right\} \\
& \times 2 \varepsilon\left(1+\frac{n-2}{3(n-1)} \varepsilon+\cdots\right)+\frac{1}{2} \varepsilon^{3}\left[\frac{4(n-2)}{3(n-1)}+\cdots\right] \times O(1),
\end{aligned}
$$

which implies $\rho\left(1-\varepsilon, x_{1}\right)>0$ for sufficiently small $\varepsilon>0$ and $\rho\left(x, x_{1}\right) \backslash$ near $x=1$, because

by Lemma 7.1 in (III).

$$
\lambda(1)-\tilde{\lambda}\left(x_{1}\right)>0
$$

This lemma corresponds to (1.16) for $n=2$.

Next, we get 


$$
g\left(x_{0}\right):=\rho\left(x_{0}, x_{1}\right)=\int_{x_{0}}^{x_{1}} \frac{N\left(x, x_{1}\right) d x}{(x-1)^{4}(n-x)^{n+1 / 2}}
$$

and want to show that $g\left(x_{0}\right)>0$ which corresponds to (1.17) for $n=2$. From the evaluation of $N\left(t, x_{1}\right)$ in the proof of Lemma 2.1, we can easily see that

$$
\lim _{x_{0} \rightarrow 1} g\left(x_{0}\right)=0 \text {. }
$$

From (2.10), we have

$$
\begin{aligned}
g^{\prime}\left(x_{0}\right)= & \frac{\partial x_{1}}{\partial x_{0}} \frac{N\left(x_{1}, x_{1}\right)}{\left(x_{1}-1\right)^{4}\left(n-x_{1}\right)^{n+1 / 2}}-\frac{N\left(x_{0}, x_{1}\right)}{\left(1-x_{0}\right)^{4}\left(n-x_{0}\right)^{n+1 / 2}} \\
& +\frac{\partial x_{1}}{\partial x_{0}} \int_{x_{0}}^{x_{1}} \frac{1}{(x-1)^{4}(n-x)^{n+1 / 2}} \frac{\partial N\left(x, x_{1}\right)}{\partial x_{1}} d x \\
= & -\frac{1-x_{0}}{x_{0}\left(n-x_{0}\right)} \cdot \frac{x_{1}\left(n-x_{1}\right)}{x_{1}-1} \cdot \frac{N\left(x_{1}, x_{1}\right)}{\left(x_{1}-1\right)^{4}\left(n-x_{1}\right)^{n+1 / 2}} \\
& -\frac{N\left(x_{0}, x_{1}\right)}{\left(1-x_{0}\right)^{4}\left(n-x_{0}\right)^{n+1 / 2}}-\frac{1-x_{0}}{x_{0}\left(n-x_{0}\right)} \cdot \frac{x_{1}\left(n-x_{1}\right)}{x_{1}-1} \\
& \times \int_{x_{0}}^{x_{1}} \frac{(n-x) F_{2}(x)}{(x-1)^{4}(n-x)^{n+1 / 2}} \frac{\left(x_{1}-1\right)\left\{n+(n-1) x_{1}\right\}}{(n-1) x_{1}^{2}\left(n-x_{1}\right)} d x
\end{aligned}
$$

by means of

$$
\frac{d \tilde{\lambda}(x)}{d x}=-\frac{(x-1)\{n+(n-1) x\}}{(n-1) x^{2}(n-x)} .
$$

Since we have

$$
\begin{gathered}
\frac{1-x_{0}}{x_{0}\left(n-x_{0}\right)} \cdot \frac{x_{1}\left(n-x_{1}\right)}{x_{1}-1} \cdot \frac{1}{\left(x_{1}-1\right)^{4}\left(n-x_{1}\right)^{n+1 / 2}} \\
=\frac{1-x_{0}}{x_{0}^{2}\left(n-x_{0}\right)^{n}} \cdot \frac{x_{1}^{2}}{\left(x_{1}-1\right)^{5}\left(n-x_{1}\right)^{1 / 2}}
\end{gathered}
$$

by $x_{0}\left(n-x_{0}\right)^{n-1}=x_{1}\left(n-x_{1}\right)^{n-1}$, we obtain

$$
\begin{aligned}
g^{\prime}\left(x_{0}\right)= & -\frac{1-x_{0}}{x_{0}^{2}\left(n-x_{0}\right)^{n}}\left[\frac{x_{1}^{2} N\left(x_{1}, x_{1}\right)}{\left(x_{1}-1\right)^{5} \sqrt{n-x_{1}}}+\frac{x_{0}^{2} N\left(x_{0}, x_{1}\right)}{\left(1-x_{0}\right)^{5} \sqrt{n-x_{0}}}\right] \\
& -\frac{1-x_{0}}{(n-1) x_{0}\left(n-x_{0}\right)} \frac{n+(n-1) x_{1}}{x_{1}} \int_{x_{0}}^{x_{1}} \frac{F_{2}(x) d x}{(x-1)^{4}(n-x)^{n-1 / 2}}
\end{aligned}
$$

i. e.

$$
\begin{aligned}
g^{\prime}\left(x_{0}\right)= & -\frac{1-x_{0}}{x_{0}^{2}\left(n-x_{0}\right)^{n}} V\left(x_{0}, x_{1}\right) \\
& -\frac{1-x_{0}}{(n-1) x_{0}\left(n-x_{0}\right)} \frac{n+(n-1) x_{1}}{x_{1}} \int_{x_{0}}^{x_{1}} \frac{F_{2}(x) d x}{(x-1)^{4}(n-x)^{n-1 / 2}} .
\end{aligned}
$$


LEMMA 2.2. When $n \geqq 2.4$, we have

$$
g^{\prime}\left(x_{0}\right)<0 \quad \text { for } 0<x_{0}<1 .
$$

Proof. Since we proved the inequality $V\left(x_{0}, x_{1}\right)>0$ for $n \geqq 84$ in (IV), for $16 \leqq n \leqq 84$ in (V), for $9.7 \leqq n \leqq 16$ in (VI), for $5 \leqq n \leqq 9.7$ in (VII), for $4.5 \leqq n \leqq 5$ in (VIII), for $2.4 \leqq n \leqq 4.5$ in (IX) and we have $F_{2}(x)>0$ for $0<x<n, x \neq 1$, with $n \geqq 2$ by Proposition 1 in (II), we obtain

$$
g^{\prime}\left(x_{0}\right)<0 \quad \text { for } 0<x_{0}<1 \text { with } n \geqq 2.4 .
$$

From (2.12), we see that $g^{\prime}\left(x_{0}\right)<0$ is equivalent to

$$
-V\left(x_{0}, x_{1}\right)<\frac{\left\{n+(n-1) x_{1}\right\}\left(n-x_{1}\right)^{n-1}}{n-1} \int_{x_{0}}^{x_{1}} \frac{F_{2}(x) d x}{(x-1)^{4}(n-x)^{n-1 / 2}}
$$

and so

$$
\begin{aligned}
\eta\left(x_{0}, n\right) & -\eta\left(x_{1}, n\right)<\frac{x_{0}^{2} \sqrt{n-x_{0}} F_{2}\left(x_{0}\right)}{\left(1-x_{0}\right)^{5}}\left\{\tilde{\lambda}\left(x_{0}\right)-\tilde{\lambda}\left(x_{1}\right)\right\} \\
& +\left(n-x_{1}\right)^{n-1}\left(x_{1}+\frac{n}{n-1}\right) \int_{x_{0}}^{x_{1}} \frac{F_{2}(x) d x}{(x-1)^{4}(n-x)^{n-1 / 2}} .
\end{aligned}
$$

By Lemma 7.1 in (III) and Lemma 2.1 in (IV), we have

$$
\tilde{\lambda}\left(x_{0}\right)-\tilde{\lambda}\left(x_{1}\right)=\frac{1}{n-1} \int_{x_{0}}^{x_{1}} \frac{(x-1)\{n+(n-1) x\} d x}{x^{2}(n-x)}
$$

and

$$
\eta\left(x_{1}, n\right)-\eta\left(x_{0}, n\right)=\frac{1}{2(n-1)} \int_{x_{0}}^{x_{1}} \frac{\left\{B \tilde{Q}_{3}(x)-(n-x)^{n-1} \tilde{Q}_{4}(x)\right\} d x}{(x-1)^{4} \sqrt{n-x}}
$$

where

$$
\begin{gathered}
\tilde{Q}_{3}(z)=\left(4 n^{2}+2 n-3\right) z^{3}-8(n-1)\left(n^{2}+5 n-3\right) z^{2} \\
+3\left(16 n^{3}-40 n^{2}+24 n-3\right) z+2 n(4 n-1)(4 n-3), \\
\tilde{Q}_{4}(z)=(n-1)(2 n-1)(2 n-3) z^{4}+(2 n-3)\left(2 n^{2}-13 n+8\right) z^{3} \\
+3\left(8 n^{3}-26 n^{2}+21 n-3\right) z^{2}+n\left(28 n^{2}-52 n+15\right) z+6 n^{2}(2 n-1) .
\end{gathered}
$$

Using these in the above inequality and introducing

$$
\begin{aligned}
G\left(x, x_{0}, n\right):= & \frac{B \tilde{Q}_{3}(x)-(n-x)^{n-1} \tilde{Q}_{4}(x)}{2(n-1)(x-1)^{4} \sqrt{n-x}} \\
& +\frac{x_{0}^{2} \sqrt{n-x_{0}} F_{2}\left(x_{0}\right)}{\left(1-x_{0}\right)^{5}} \frac{x-1}{x^{2}(n-x)}\left(x+\frac{n}{n-1}\right) \\
& +\left(n-x_{1}\right)^{n-1}\left(x_{1}+\frac{n}{n-1}\right) \frac{F_{2}(x)}{(x-1)^{4}(n-x)^{n-1 / 2}},
\end{aligned}
$$


$g^{\prime}\left(x_{0}\right)<0$ is equivalent to

$$
J:=\int_{x_{0}}^{x_{1}} G\left(x, x_{0}, n\right) d x>0 .
$$

We can prove that

$$
\int_{x_{0}}^{x_{1}} G\left(x, x_{0}, 2\right) d x>0 \quad \text { for } 0<x_{0}<1 .
$$

Now looking over the expression of $G\left(x, x_{0}, n\right)$, we divide the above integral $J$ into the three parts as follows:

$$
\begin{gathered}
J=J_{1}+J_{2}+J_{3}, \\
J_{1}=\frac{x_{0}^{2} \sqrt{n-x_{0}} F_{2}\left(x_{0}\right)}{\left(1-x_{0}\right)^{5}} \int_{x_{0}}^{x_{1}} \frac{x-1}{x^{2}(n-x)}\left(x+\frac{n}{n-1}\right) d x \\
=\frac{x_{0}^{2} \sqrt{n-x_{0}} F_{2}\left(x_{0}\right)}{\left(1-x_{0}\right)^{5}}\left\{\log \frac{n-x_{0}}{n-x_{1}}-\frac{1}{n-1}\left(\frac{1}{x_{0}}-\frac{1}{x_{1}}\right)\right\} . \\
J_{2}=\frac{1}{2(n-1)} \int_{x_{0}}^{x_{1}} \frac{1}{(x-1)^{4} \sqrt{n-x}}\left\{B \tilde{Q}_{3}(x)-(n-x)^{n-1} \tilde{Q}_{4}(x)\right\} d x \\
=\eta\left(x_{1}, n\right)-\eta\left(x_{0}, n\right), \\
J_{3}=\left(n-x_{1}\right)^{n-1}\left(x_{1}+\frac{n}{n-1}\right) \int_{x_{0}}^{x_{1}} \frac{F_{2}(x) d x}{(x-1)^{4}(n-x)^{n-1 / 2}} .
\end{gathered}
$$

In order to make easy the handling of $J_{2}$ we use the parameter $y=x-1$, then we obtain by Lemma 2.2 in (IX)

$$
\tilde{Q}_{3}(1+y)=\sum_{i=1}^{3} a_{i}(n) y^{\imath}
$$

where

and

$$
\begin{aligned}
& a_{0}=72 n^{3}-180 n^{2}+144 n-36=36(n-1)^{2}(2 n-1), \\
& a_{1}=32 n^{3}-172 n^{2}+206 n-66=2(n-1)\left(16 n^{2}-70 n+33\right), \\
& a_{2}=-8 n^{3}-20 n^{2}+70 n-33, \quad a_{3}=4 n^{2}+2 n-3
\end{aligned}
$$

$$
\tilde{Q}_{4}(1+y)=\sum_{i=0}^{4} b_{i}(n) y^{2}
$$

where

$$
\begin{aligned}
& b_{0}=72 n^{3}-180 n^{2}+144 n-36=36(n-1)^{2}(2 n-1), \\
& b_{1}=104 n^{3}-352 n^{2}+350 n-102=2(n-1)\left(52 n^{2}-124 n+51\right), \\
& b_{2}=60 n^{3}-246 n^{2}+294 n-99=3\left(20 n^{3}-82 n^{2}+98 n-33\right),
\end{aligned}
$$




$$
\begin{aligned}
& b_{3}=20 n^{3}-80 n^{2}+99 n-36, \\
& b_{4}=4 n^{3}-12 n^{2}+11 n-3=(n-1)\left(4 n^{2}-8 n+3\right) .
\end{aligned}
$$

Analogously, we have for $P_{2}(x)$ and $P_{3}(x)$

$$
\left\{\begin{array}{l}
P_{2}(1+y)=12(n-1)^{2}-2(n-1)(2 n+5) y+(2 n+1) y^{2}, \\
P_{3}(1+y)=12(n-1)^{2}+2(n-1)(4 n-11) y+\left(2 n^{2}-10 n+11\right) y^{2}-(n-1) y^{3} .
\end{array}\right.
$$

Regarding $J_{2}$ and $J_{3}$, we have the following Lemmas 2.3-Lemma 2.6, which will be proved in another paper ([24]).

LEMMA 2.3. Regarding $\tilde{Q}_{3}(1+y)$ we have the following:

i) $\widetilde{Q}_{3}(1+y)$ is $\nearrow$ in $-1<y<0$ and $\searrow$ in $0<y<n-1$ with $2 \leqq n \leqq 3.5$, and $>0$ for $-1 \leqq y \leqq n-1$ with $2 \leqq n \leqq(5+\sqrt{13}) / 4$,

and $>0$ for $-1 \leqq y \leqq 1$ and $<0$ at $y=n-1$ with $(5+\sqrt{13}) / 4<n \leqq 3.5$;

ii) $\tilde{Q}_{3}(1+y) / y^{4} \sqrt{1-\frac{y}{n-1}}$ is $\nearrow$ in $-1<y<0$

and positive for $-1 \leqq y<0$, tends to $+\infty$ as $y \rightarrow-0$ with $2 \leqq n \leqq 2.65$, and $\searrow ~$ in $0<y<n-1$, positive for $0<y<n-1$,

tends to $+\infty$ as $y \rightarrow+0$ or $y \rightarrow n-1$ with $2 \leqq n<(5+\sqrt{13}) / 4$, and $\searrow$ in $0<y<n-1$, positive for $0<y \leqq 1$,

tends to $+\infty$ as $y \rightarrow+0$ and $-\infty$ as $y \rightarrow n-1$. with $(5+\sqrt{13}) / 4<n \leqq 2.65$ (see Fig. 1).

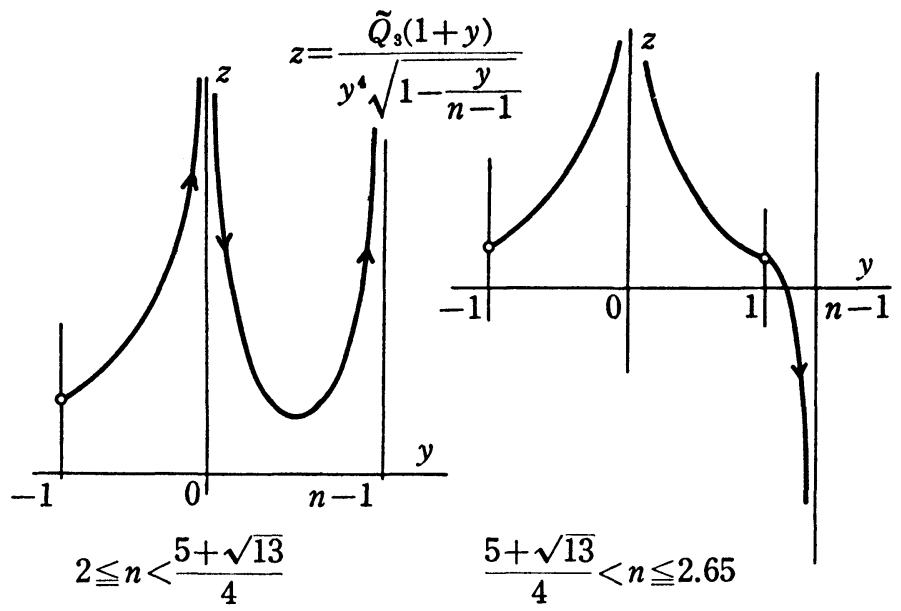

Fig. 1.

LEMMA 2.4. Regarding $\tilde{Q}_{4}(1+y)$ we have the following:

i) $\tilde{Q}_{4}(1+y)>0$ for $-1 \leqq y \leqq n-1$ with $n \geqq 2$; 
ii) $\frac{1}{y^{4}}\left(1-\frac{y}{n-1}\right)^{n-3 / 2} \widetilde{Q}_{4}(1+y)$ is $\nearrow$ in $-1<y<0$, \in $0<y<n-1$, positive for $-1 \leqq y \leqq n-1, \neq 0$, tends to $+\infty$ as $y \rightarrow 0$, with $2 \leqq n \leqq 2.5$.

LEMMA 2.5. Regarding $P_{2}(1+y)$ we have the following:

i) as a quadratic polynomial of $y$, its $y$-coordinate of symmetric axis $(n-1)(2 n+5) /(2 n+1)>n-1$ with $n \geqq 2$, it takes its values $12(n-1)^{2}$ at $y=0$ and $(n-1)^{2}(3-2 n)$ at $y=n-1$;

ii) $\frac{P_{2}(1+y)}{y^{4}\left(1-\frac{y}{n-1}\right)^{n-1 / 2}}$ is $\nearrow$ in $-1<y<0$, positive for $-1 \leqq y<0$, tends to $+\infty$ as $y \rightarrow-0$, and $\searrow$ in $0<y<n-1$ with $n \geqq 2$.

LEMMA 2.6. Regarding $P_{3}(1+y)$ we have the following: 2.722 ;

i) $P_{3}(1+y)$ is $\searrow$ in $-1<y<n-1$, positive for $-1 \leqq y \leqq n-1$ with $2 \leqq n \leqq$

ii) $\frac{P_{3}(1+y)}{y^{4} \sqrt{1-\frac{y}{n-1}}}$ is $\nearrow$ in $-1<y<0$, tends to $+\infty$ as $y \rightarrow-0$,

and $\searrow \nearrow$ in $0<y<n-1$, tends to $+\infty$ as $y \rightarrow+0$ or $y \rightarrow n-1$ with $2 \leqq n \leqq 2.64$.

Now, regarding the integral $J_{2}$ and $J_{3}$ we have

$$
J_{2}=\frac{(n-1)^{n-5 / 2}}{2}\left[\int_{-1}^{n-1} \frac{\tilde{Q}_{3}(1+y) d y}{y^{4} \sqrt{1-\frac{y}{n-1}}}-\int_{-1}^{n-1} \frac{1}{y^{4}}\left(1-\frac{y}{n-1}\right)^{n-3 / 2} \tilde{Q}_{4}(1+y) d y\right]
$$

and

$$
J_{3}=\frac{\left(n-x_{1}\right)^{n-1}}{\sqrt{n-1}}\left(x_{1}+\frac{n}{n-1}\right)\left[-\int_{-1}^{n-1} \frac{P_{2}(1+y) d y}{y^{4}\left(1-\frac{y}{n-1}\right)^{n-1 / 2}}+\int_{-1}^{n-1} \frac{P_{3}(1+y) d y}{y^{4} \sqrt{1-\frac{y}{n-1}}}\right]
$$

in which the integrands in the brackets all tend to $+\infty$ as $y \rightarrow 0$. Therefore in order to evaluate $J_{2}$ and $J_{3}$ we need the following lemmas, which will be proved also in [24].

LEMMA 2.7. Regarding $J_{2}$ we have

$$
\begin{aligned}
\int_{0.99}^{1.01} & \frac{1}{(x-1)^{4} \sqrt{n-x}}\left\{B \tilde{Q}_{3}(x)-(n-x)^{n-1} \tilde{Q}_{4}(x)\right\} d x \\
& >\frac{4}{3}(n-1)^{n+1 / 2}\left(b^{\prime}-b\right)+\frac{1}{3}(n-1)^{n-2} n(2 n-1)\left(n^{2}-n-3\right) \\
& \times(\sqrt{n-0.99}-\sqrt{n-1.01})+\frac{4}{3}(n-1)^{n-1} \\
\quad & \times\left\{\sqrt{n-0.99}\left(n-\frac{201}{200}\right) b-\sqrt{n-1.01}\left(n-\frac{199}{200}\right) b^{\prime}\right\}
\end{aligned}
$$


where

$b=b_{5}$ for $2 \leqq n \leqq 2.24, b=b_{6}$ for $2.24<n \leqq 2.40$ and $b^{\prime}=b_{7}$,

$$
\begin{aligned}
& b_{5}=b_{4}-\frac{n-2}{2(n-1)} b_{3}-\frac{(n-2)(3-n)}{\mathfrak{6}(n-1)^{2}}\left(\frac{n-1}{n-0.99}\right)^{4-n} b_{2} \\
& -\frac{(n-2)(3-n)(4-n)}{24(n-1)^{3}}\left(b_{1}-\frac{1}{50} b_{2}+\frac{3}{10000} b_{3}-\frac{1}{250000} b_{4}\right) \\
& -\frac{(n-2)(3-n)(4-n)(5-n)}{120(n-1)^{4}} b_{0} \\
& b_{6}=b_{4}-\frac{n-2}{2(n-1)} b_{3}-\frac{(n-2)(3-n)}{6(n-1)^{2}} b_{2}-\frac{(n-2)(3-n)(4-n)}{24(n-1)^{3}} \\
& \times 1.00016 \times b_{1}-\frac{(n-2)(3-n)(4-n)(5-n)}{120(n-1)^{4}} b_{0}, \\
& b_{7}=\left(\frac{n-1.01}{n-1}\right)^{n-2} b_{4}-\frac{n-2}{2(n-1)}\left(\frac{n-1}{n-1.01}\right)^{3-n}\left(b_{3}+\frac{1}{25} b_{4}\right) \\
& -\frac{(n-2)(3-n)}{6(n-1)^{2}}\left(\frac{n-1}{n-1.01}\right)^{4-n}\left(b_{2}+\frac{3}{100} b_{3}+\frac{3}{5000} b_{4}\right) \times h \\
& -\frac{(n-2)(3-n)(4-n)}{24(n-1)^{3}}\left(\frac{n-1}{n-1.01}\right)^{5-n} k \times b_{1} \\
& -\frac{(n-2)(3-n)(4-n)(5-n)}{120(n-1)^{4}}\left(\frac{n-1}{n-1.01}\right)^{6-n} \\
& \times\left(b_{0}+\frac{1}{10^{2}} b_{1}+\frac{1}{10^{4}} b_{2}+\frac{1}{10^{6}} b_{3}+\frac{1}{10^{8}} b_{4}\right), \\
& \left\{\begin{array}{c}
k=1 \text { for } 2 \leqq n \leqq 2.2413 \cdots, \\
1.002 \text { for } 2.2413 \cdots<n<2.32, \\
1.0034 \text { for } 2.32 \leqq n \leqq 2.4,
\end{array}\right. \\
& \left\{\begin{array}{c}
h=\left(\frac{n-1.01}{n-1}\right)^{4-n} \text { for } 2 \leqq n<2.2413 \cdots, \\
1 \text { for } 2.2413 \cdots<n \leqq 2.4 .
\end{array}\right.
\end{aligned}
$$

LEMMA 2.8. Reading $J_{3}$ we have

$$
\begin{gathered}
\int_{0.99}^{1.01} \frac{F_{2}(x) d x}{(1-x)^{4}(n-x)^{n-1 / 2}}>\frac{n\left(n^{2}-n+1\right)}{21 \sqrt{n-1}}\left\{1-\left(\frac{n-1}{n-0.99}\right)^{7 / 2}\right\} \\
+\frac{n}{24(n-1)^{5 / 2}(n-3 / 2)}\left\{\left(\frac{n-1}{n-1.01}\right)^{n-3 / 2}-1\right\} \times b_{10},
\end{gathered}
$$


where

$$
\begin{aligned}
b_{10}= & 3.9202 n^{4}-11.780001 n^{3}+15.680498 n^{2} \\
& -11.740299 n+3.919002 \text { for } n \geqq 2 .
\end{aligned}
$$

PROPOSITION 2. Regarding $\rho\left(x, x_{1}\right)$ defined by (2.9) we have

with $n \geqq 2$.

$$
\rho\left(x_{0}, x_{1}\right)>0 \quad \text { for } 0<x_{0}<1<x_{1}<n, x_{1}=X_{n}\left(x_{0}\right)
$$

Proof. By (2.11), it is sufficient to show

$$
g^{\prime}\left(x_{0}\right)<0 \quad \text { for } 0<x_{0}<1,
$$

which is held by Lemma 2.1 for $n \geqq 2.4$. For $2 \leqq n \leqq 2.4$, we proved this inequality by showing that (2.17) holds by means of numerical evaluation of $J$ by computors in which we computed $J_{1}, J_{2}$ and $J_{2}$ for $0.01 \leqq x_{0} \leqq 0.99$ and $2 \leqq n \leqq 2.41$ with step $1 / 100$ and obtained $J>0$ for these values of $x_{0}$ and $n$, from which we see that this inequality holds for $0<x_{0}<1$ and $2 \leqq n \leqq 2.4$ in general, by taking the properties of $J_{1}, J_{2}, J_{3}$ by Lemma 2.3-Lemma 2.8 into consideration.

Q.E.D.

As the next step, we wish to prove $\rho\left(x, x_{1}\right)>0$ for $x_{0}<x<1$, which corresponds to (1.18) in the proof of Proposition 1. If this inequality does not hold, then there exist $\xi_{1}, \xi_{2}$ such that $x_{0}<\xi_{1} \leqq \xi_{2}<1$,

and

$$
\rho\left(\xi_{1}, x_{1}\right)=\rho\left(\xi_{2}, x_{1}\right)=0,
$$

$$
\frac{\partial \rho\left(x, x_{1}\right)}{\partial x} \leqq 0 \text { at } x=\xi_{1} \text { and } \frac{\partial \rho\left(x, x_{1}\right)}{\partial x} \geqq 0 \text { at } x=\xi_{2}
$$

by Lemma 2.1 and Proposition 2. From (2.1) and (2.9) we obtain

$$
\begin{aligned}
\frac{\partial \rho\left(x, x_{1}\right)}{\partial x} & =\frac{N\left(X, x_{1}\right)}{(X-1)^{4}(n-X)^{n+1 / 2}} \frac{\partial X}{\partial x}-\frac{N\left(x, x_{1}\right)}{(1-x)^{4}(n-x)^{n+1 / 2}} \\
& =-\frac{1-x}{x(n-x)}\left[\frac{X N\left(X, x_{1}\right)}{(X-1)^{5}(n-X)^{n-1 / 2}}+\frac{x N\left(x, x_{1}\right)}{(1-x)^{5}(n-x)^{n-1 / 2}}\right] \\
& =-\frac{1-x}{x^{2}(n-x)^{2}}\left[\frac{X^{2} N\left(X, x_{1}\right)}{(X-1)^{5} \sqrt{n-X}}+\frac{x^{2} N\left(x, x_{1}\right)}{(1-x)^{5} \sqrt{n-x}}\right],
\end{aligned}
$$

i.e.

$$
\frac{\partial p\left(x, x_{1}\right)}{\partial x}=-\frac{1-x}{x^{2}(n-x)^{n}} V\left(x, x_{1}\right) \quad \text { for } 0<x<1,
$$

by (7.10) in (III). Hence, the above inequalities for $\xi_{1}$ and $\xi_{2}$ become

$$
V\left(\xi_{1}, x_{1}\right) \geqq 0 \text { and } V\left(\xi_{2}, x_{1}\right) \leqq 0 .
$$


Therefore, if we can prove that

$$
\frac{\partial V\left(x, x_{1}\right)}{\partial x}>0 \quad \text { for } x_{0}<x<1
$$

then it implies that

and

$$
\xi_{1}=\xi_{2} \text { and } V\left(\xi_{1}, x_{1}\right)=0
$$

$$
\rho\left(x, x_{1}\right)>0 \quad \text { for } x_{0}<x<\xi_{1} \text { and } \xi_{1}<x<1,
$$

which also implies $I>0$ by (2.7) and (2.9). In the following we shall try to prove (2.26).

§3. $\partial V\left(x, x_{1}\right) / \partial x$.

LEMMA 3.1. Setting $x=1-t$ and $X_{n}(x)=1+s$ near $x=1$, we have

$$
s=t+\frac{2(n-2)}{3(n-1)} t^{2}+\frac{4(n-2)^{2}}{9(n-1)^{2}} t^{3}+\cdots .
$$

Proof. From $x(n-x)^{n-1}=X(n-X)^{n-1}$, we have

$$
(1-t)\left(1+\frac{t}{n-1}\right)^{n-1}=(1+s)\left(1-\frac{s}{n-1}\right)^{n-1}
$$

i. e.

$$
\begin{aligned}
1- & \frac{n}{2(n-1)} t^{2}-\frac{n(n-2)}{3(n-1)^{2}} t^{3}-\frac{n(n-2)(n-3)}{8(n-1)^{3}} t^{4}-\cdots \\
& =1-\frac{n}{2(n-1)} s^{2}+\frac{n(n-2)}{3(n-1)^{2}} s^{3}-\frac{n(n-2)(n-3)}{8(n-1)^{3}} s^{4}+\cdots
\end{aligned}
$$

By (8.12) in (III), we can put

$$
s=t+\frac{2(n-2)}{3(n-1)} t^{2}+b t^{3}+\cdots
$$

and substituting this expression into the above equality we obtain easily

$$
b=\frac{4(n-2)^{2}}{9(n-1)^{2}} \text {. }
$$

Q.E.D.

LEMMA 3.2. Near $x=1$, we have

$$
\begin{aligned}
& \frac{1}{B} V\left(x, x_{1}\right)=\frac{n\left(n^{2}-n+1\right) C}{3 \sqrt{n-1}(1-x)}-\frac{n(n-2)\left(n^{2}-n+1\right) C}{9(n-1)^{3 / 2}} \\
& -\frac{n\left\{144(2 n-1)+\left(n^{2}-n+1\right)\left(32 n^{2}-128 n+137\right) C\right\}}{216(n-1)^{5 / 2}}(1-x)+\cdots,
\end{aligned}
$$


where $C=\log (n-1)+1-\tilde{\lambda}\left(x_{1}\right)=\lambda(1)-\tilde{\lambda}\left(x_{1}\right)>0$.

Proof. Setting $x=1-t$ and $X=X_{n}(x)=1+s$, we obtain

$$
\begin{aligned}
\frac{1}{B} F_{2}(1-t)= & \frac{n\left(n^{2}-n+1\right)}{6(n-1)} t^{4}+\frac{n(n-2)\left(6 n^{2}-7 n+7\right)}{60(n-1)^{2}} t^{5} \\
& +\frac{n(n-2)(n-3)\left(4 n^{2}-6 n+5\right)}{120(n-1)^{3}} t^{6}+\cdots
\end{aligned}
$$

by an analogous computation as the one in $\S 8$ of (III), and

$$
\begin{aligned}
& \frac{1}{B} f_{0}(1-t)=-\frac{n(2 n-1)}{6(n-1)} t^{3}-\frac{n(n-2)(3 n-1)}{12(n-1)^{2}} t^{4}+\cdots, \\
& \frac{1}{B}\{B-\phi(1-t)\}=\frac{n}{2(n-1)} t^{2}+\frac{n(n-2)}{3(n-1)^{2}} t^{3}+\cdots, \\
& \frac{x^{2}}{\sqrt{n-x}}=\frac{1}{\sqrt{n-1}}\left\{1-\frac{4 n-3}{2(n-1)} t+\frac{8 n^{2}-8 n+3}{8(n-1)^{2}} t^{2}+\cdots\right\}, \\
& \lambda(1-t)=\log (n-1)+1+\frac{1}{2(n-1)^{2}} t^{2}-\frac{2}{3(n-1)^{3}} t^{3}+\cdots .
\end{aligned}
$$

Hence, we obtain

$$
\begin{aligned}
\frac{x^{2} N}{\left.B(1-x)^{5} \sqrt{ } x_{1}\right)}=\frac{1}{\sqrt{n-1}}\left\{1-\frac{4 n-3}{2(n-1)} t+\frac{8 n^{2}-8 n+3}{8(n-1)^{2}} t^{2}+\cdots\right\} \\
\quad \times\left[\frac { n - 1 + t } { t } \left\{\frac{n\left(n^{2}-n+1\right)}{6(n-1)}+\frac{n(n-2)\left(6 n^{2}-7 n+7\right)}{60(n-1)^{2}} t\right.\right. \\
\left.\quad+\frac{n(n-2)(n-3)\left(4 n^{2}-6 n+5\right)}{120(n-1)^{3}} t^{2}+\cdots\right\}\left\{C+\frac{1}{2(n-1)^{2}} t^{2}\right. \\
\left.\quad-\frac{2}{3(n-1)^{2}} t^{3}+\cdots\right\}-\frac{n(2 n-1)}{2(n-1)}-\frac{n(n-2)(3 n-1)}{4(n-1)^{2}} t+\cdots \\
\left.\quad+2 n\left\{\frac{n}{2(n-1)}+\frac{n(n-2)}{3(n-1)^{2}} t+\cdots\right\}\right] \\
=\frac{1}{\sqrt{n-1}-\left\{1-\frac{4 n-3}{2(n-1)} t+\frac{8 n^{2}-8 n+3}{8(n-1)^{2}} t^{2}+\cdots\right\}} \\
\quad \times\left[\frac{n\left(n^{2}-n+1\right) C}{6 t}+\frac{n\left(6 n^{3}-9 n^{2}+11 n-4\right) C}{60(n-1)}+\frac{n}{2(n-1)}\right. \\
\left.+\left(\frac{n(4 n-5)}{12(n-1)^{2}}+\frac{n(n-2)\left(4 n^{3}-6 n^{2}+9 n-1\right) C}{120(n-1)^{2}}\right) t+\cdots\right]
\end{aligned}
$$

i. e. 


$$
\begin{aligned}
& \frac{\sqrt{n-1} x^{2} N\left(x, x_{1}\right)}{B(1-x)^{5} \sqrt{n-x}}=\frac{n\left(n^{2}-n+1\right) C}{6 t}+\frac{n}{2(n-1)} \\
& -\frac{n\left(14 n^{3}-26 n^{2}+24 n-11\right) C}{60(n-1)}-\left\{\frac{n(2 n-1)}{3(n-1)^{2}}+\frac{n\left(n^{2}-n+1\right) C}{48(n-1)^{2}}\right\} t+\cdots
\end{aligned}
$$

We obtain analogously

$$
\begin{aligned}
& \frac{\sqrt{n-1} X^{2} N\left(X, x_{1}\right)}{B(X-1)^{5} \sqrt{n-X}}=\frac{n\left(n^{2}-n+1\right) C}{6 s}-\frac{n}{2(n-1)} \\
& +\frac{n\left(14 n^{3}-26 n^{2}+24 n-11\right) C}{60(n-1)}-\left\{\frac{n(2 n-1)}{3(n-1)^{2}}+\frac{n\left(n^{2}-n+1\right) C}{48(n-1)^{2}}\right\} s+\cdots
\end{aligned}
$$

Therefore, using (3.1) and (2.1) we obtain

$$
\begin{aligned}
\frac{\sqrt{n-1}}{B} & V\left(x, x_{1}\right)=\frac{n\left(n^{2}-n+1\right) C}{6}\left(\frac{1}{t}+\frac{1}{s}\right) \\
& -\left\{\frac{n(2 n-1)}{3(n-1)^{2}}+\frac{n\left(n^{2}-n+1\right) C}{48(n-1)^{2}}\right\}(t+s)+\cdots \\
= & \frac{n\left(n^{2}-n+1\right) C}{6 t}\left\{2-\frac{2(n-2)}{3(n-1)} t-\frac{8(n-2)^{2}}{9(n-1)^{2}} t^{2}+\cdots\right\} \\
& -\left\{\frac{n(2 n-1)}{3(n-1)^{2}}+\frac{n\left(n^{2}-n+1\right) C}{48(n-1)^{2}}\right\}(2 t+\cdots) \\
= & \frac{n\left(n^{2}-n+1\right) C}{3 t}-\frac{n(n-2)\left(n^{2}-n+1\right) C}{9(n-1)} \\
& -\frac{n\left\{144(2 n-1)+\left(n^{2}-n+1\right)\left(32 n^{2}-128 n+137\right) C\right\}}{216(n-1)^{2}} t+\cdots,
\end{aligned}
$$

which implies this lemma.

Q.E.D.

LEMMA 3.3. We have for $1<x_{1}<n$

i) $\lim _{x \rightarrow 1-0} V\left(x, x_{1}\right)=+\infty$ with $n>1$;

ii) $\lim _{x \rightarrow 1-0}(1-x) V\left(x, x_{1}\right)=\frac{B n\left(n^{2}-n+1\right) C}{3 \sqrt{n-1}}$ with $n>1$;

and $(1-x) V\left(x, x_{1}\right)$ is $\nearrow$ at $x=1$ with $>2$;

iii) $\lim _{x \rightarrow 1-0} \frac{\partial \rho\left(x, x_{1}\right)}{\partial x}=-\frac{n\left(n^{2}-n+1\right) C}{3(n-1)^{3 / 2}}<0$,

where $C=\lambda(1)-\tilde{\lambda}\left(x_{1}\right)=\log \frac{n-1}{n-x_{1}}-\frac{x_{1}-1^{\prime}}{(n-1) x_{1}}>0$.

Proof. i) and ii) are evident from Lemma 3.2. Next, from (2.24) we obtain 


$$
\begin{aligned}
\lim _{x \rightarrow 1-0} \frac{\partial \rho\left(x, x_{1}\right)}{\partial x} & =-\frac{1}{(n-1)^{n}} \lim _{x \rightarrow 1-0}\left\{(1-x) V\left(x, x_{1}\right)\right\} \\
& =-\frac{n\left(n^{2}-n+1\right) C}{3(n-1)^{3 / 2}}<0 .
\end{aligned}
$$

Q.E.D.

Now, we compute $\partial V\left(x, x_{1}\right) / \partial x$. Regarding the functions in (1.2), we have

$$
\begin{gathered}
\left(\frac{x^{2} \sqrt{n-x}}{(1-x)^{5}}\right)^{\prime}=\frac{x\left\{4 n+(6 n-5) x-5 x^{2}\right\}}{2(1-x)^{6} \sqrt{n-x}}, \\
F_{2}^{\prime}(x)=-2 B\left\{(2 n+1) x-\left(2 n^{2}+5 n-4\right)\right\}-(n-x)^{n-2} Q_{3}(x),
\end{gathered}
$$

where

$$
Q_{3}(x)=2 n^{2}(n+2)+n(n-13) x+2\left(n^{3}-n^{2}-n+4\right) x^{2}-(n-1)(n+2) x^{3}
$$

((3.1) and (3.2) in (VII)),

$$
\tilde{\lambda}^{\prime}(x)=-\frac{(x-1)\{n+(n-1) x\}}{(n-1) x^{2}(n-x)}
$$

(Lemma 7.1 in (III)), from which we obtain

$$
\begin{aligned}
& \left(\frac{x^{2} \sqrt{n-x} F_{2}(x)}{(1-x)^{5}}\left\{\tilde{\lambda}(x)-\tilde{\lambda}\left(x_{1}\right)\right\}\right)^{\prime} \\
& \quad=\frac{\{n+(n-1) x\} F_{2}(x)}{(n-1)(1-x)^{4} \sqrt{n-x}}+\frac{x W(x, n)\left\{\tilde{\lambda}(x)-\tilde{\lambda}\left(x_{1}\right)\right\}}{2(1-x)^{6} \sqrt{n-x}},
\end{aligned}
$$

where

$$
\begin{aligned}
W(x, n): & =-B W_{4}(x, n)+(n-x)^{n-1} W_{5}(x, n)(=W(x)), \\
W_{4}(x, n):= & 4 n(4 n-1)(4 n-3)+\left(72 n^{3}-236 n^{2}+146 n-15\right) x \\
& -\left(16 n^{3}+76 n^{2}-190 n+71\right) x^{2}+\left(16 n^{2}+14 n-33\right) x^{3} \\
& -(2 n+1) x^{4}\left(=W_{4}(x)\right), \\
W_{5}(x, n):= & 12 n^{2}(2 n-1)+3 n\left(16 n^{2}-36 n+9\right) x \\
& +3\left(12 n^{3}-50 n^{2}+52 n-5\right) x^{2}+\left(8 n^{3}-70 n^{2}+130 n-71\right) x^{3} \\
& +\left(4 n^{3}-18 n^{2}+44 n-33\right) x^{4}-(n-1)(2 n-1) x^{5}\left(=W_{5}(x)\right) .
\end{aligned}
$$

By Lemma 2.1 in (IX) we have

$$
\eta^{\prime}(x, n)=\frac{1}{2(n-1)(1-x)^{4} \sqrt{n-x}}\left\{B \tilde{Q}_{3}(x, n)-(n-x)^{n-1} \tilde{Q}_{4}(x, n)\right\}
$$




$$
\begin{aligned}
\tilde{Q}_{3}(x, n)= & 2 n(4 n-1)(4 n-3)+3\left(16 n^{3}-40 n^{2}+24 n-3\right) x \\
& -8(n-1)\left(n^{2}+5 n-3\right) x^{2}+\left(4 n^{2}+2 n-3\right) x^{3}\left(=\tilde{Q}_{3}(x)\right), \\
\tilde{Q}_{4}(x, n)= & 6 n^{2}(2 n-1)+n\left(28 n^{2}-52 n+15\right) x \\
& +3\left(8 n^{3}-26 n^{2}+21 n-3\right) x^{2}+(2 n-3)\left(2 n^{2}-13 n+8\right) x^{3} \\
& +(n-1)(2 n-1)(2 n-3) x^{4}\left(=\tilde{Q}_{4}(x)\right)
\end{aligned}
$$

and hence

$$
\begin{aligned}
V^{\prime}\left(x, x_{1}\right)= & \frac{\partial}{\partial x} V\left(x, x_{1}\right)=\frac{\{n+(n-1) x\} F_{2}(x)}{(n-1)(1-x)^{4} \sqrt{n-x}} \\
& +\frac{x W(x)\left\{\tilde{\lambda}(x)-\tilde{\lambda}\left(x_{1}\right)\right\}}{2(1-x)^{6} \sqrt{n-x}}-\frac{B \tilde{Q}_{3}(x)-(n-x)^{n-1} \tilde{Q}_{4}(x)}{2(n-1)(1-x)^{4} \sqrt{n-x}} \\
& +\left[\frac{\{n+(n-1) X\} F_{2}(X)}{(n-1)(X-1)^{4} \sqrt{n-X}}+\frac{X W(X)\left\{\tilde{\lambda}(X)-\tilde{\lambda}\left(x_{1}\right)\right\}}{2(X-1)^{6} \sqrt{n-X}}\right. \\
& \left.-\frac{B \tilde{Q}_{3}(X)-(n-X)^{n-1} \tilde{Q}_{4}(X)}{2(n-1)(X-1)^{4} \sqrt{n-X}}\right] \frac{1-x}{x(n-x)} \cdot \frac{X(n-X)}{X-1} .
\end{aligned}
$$

Carefully arranging the right hand side, we obtain the following important formula :

$$
\begin{aligned}
& \frac{x(n-x)}{1-x} \frac{\partial V\left(x, x_{1}\right)}{\partial x}=\frac{x^{2} \sqrt{n-x} W(x, n)}{2(1-x)^{7}}\left\{\tilde{\lambda}(x)-\tilde{\lambda}\left(x_{1}\right)\right\} \\
& \quad+\frac{x \sqrt{n-x S}(x, n)}{2(n-1)(1-x)^{5}}+\frac{\mid X^{2} \sqrt{n-X W}(X, n)}{2(X-1)^{7}}\left\{\tilde{\lambda}(X)-\tilde{\lambda}\left(x_{1}\right)\right\} \\
& \quad+\frac{X \sqrt{n-X} S(X, n)}{2(n-1)(X-1)^{5}}
\end{aligned}
$$

where $X=X_{n}(x)$ and

$$
\begin{aligned}
S(x, n):= & -B S_{3}(x, n)+(n-x)^{n-1} S_{4}(x, n)(=S(x)), \\
S_{3}(x, n):= & 4 n(4 n-1)(4 n-3)+3\left(24 n^{3}-68 n^{2}+42 n-5\right) x \\
& -2\left(8 n^{3}+20 n^{2}-15 n+20\right) x^{2}+\left(8 n^{2}-5\right) x^{3}\left(=S_{3}(x)\right), \\
S_{4}(x, n):= & 12 n^{2}(2 n-1)+3 n\left(16 n^{2}-32 n+9\right) x \\
& +3\left(12 n^{3}-42 n^{2}+37 n-5\right) x^{2}+\left(8 n^{3}-52 n^{2}+87 n-40\right) x^{3} \\
& +(n-1)\left(4 n^{2}-10 n+5\right) x^{4} .
\end{aligned}
$$

Looking over the right hand side of (3.8), we see that if we have

$$
W(x, n)>0 \quad \text { for } 0<x<n, x \neq 1,
$$


then it is increasing with respect to $x_{1}$, since

$$
\tilde{\lambda}^{\prime}\left(x_{1}\right)=-\frac{\left(x_{1}-1\right)\left\{n+(n-1) x_{1}\right\}}{(n-1) x_{1}^{2}\left(n-x_{1}\right)}<0 \quad \text { for } 1<x_{1}<n .
$$

Furthermore, if (3.12) holds, we shall obtain

$$
\frac{\partial V\left(x, x_{1}\right)}{\partial x}>0
$$

by proving that

$$
\begin{aligned}
\Psi(x, n):=- & \frac{x^{2} \sqrt{n-x W}(x, n)}{(1-x)^{2}}\left\{\tilde{\lambda}\left(X_{n}(x)\right)-\tilde{\lambda}(x)\right\} \\
& +\frac{x \sqrt{n-x} S(x, n)}{(n-1)(1-x)^{5}}+\frac{X \sqrt{n-X} S(X, n)}{(n-1)(X-1)^{5}}>0 \text { for } 0<x<1 .
\end{aligned}
$$

Remark. By Lemma 4.1 in (IX), $\tilde{\lambda}\left(X_{n}(x)\right)-\tilde{\lambda}(x)$ is decreasing in $0<x<1$ and positive there and

$$
\lim _{x \rightarrow+0} \tilde{\lambda}\left(X_{n}(x)\right)-\tilde{\lambda}(x)=+\infty, \quad \tilde{\lambda}\left(X_{n}(1)\right)-\tilde{\lambda}(1)=0 .
$$

$\S 4$. Some properties of $W(x, n)$.

LEMMA 4.1. $W(0, n)>0$ for $n \geqq 2$.

Proof. From (3.5), (3.6) and (3.7) we have

$$
W(0, n)=-4 B n(4 n-1)(4 n-3)+12 n^{n+1}(2 n-1),
$$

and we shall prove the following inequality:

$$
e_{n-1}:=\left(\frac{n}{n-1}\right)^{n-1}>h(n):=\frac{(4 n-1)(4 n-3)}{3 n(2 n-1)} \quad \text { for } n \geqq 2 \text {. }
$$

We see easily that (4.1) holds for $n=2$ and as $n \rightarrow \infty$ and $e_{n-1}$ and $h(n)$ are increasing in $2<n<\infty$. If (4.1) does not hold, there exist $n_{1}$ and $n_{2}$ such that $2<n_{1} \leqq n_{2}<\infty$,

$$
\begin{aligned}
& e_{n-1}=h(n) \quad \text { at } n=n_{1}, n_{2} \text { and } \\
& \log \frac{n}{n-1}-\frac{1}{n} \leqq \frac{h^{\prime}}{h}=\frac{32 n^{2}-32 n+10}{(2 n-1)(4 n-1)(4 n-3)}-\frac{1}{n} \quad \text { at } n=n_{1} \\
& \geqq \quad \text { at } n=n_{2} .
\end{aligned}
$$

On the other hand, since we have

$$
\log \frac{n}{n-1}=\frac{1}{n}+\frac{1}{2 n^{2}}+\frac{1}{3 n^{3}}+\cdots<\frac{6 n^{2}-3 n-1}{6 n^{2}(n-1)} \quad \text { for } n>1,
$$


we consider the inequality :

$$
\frac{6 n^{2}-3 n-1}{6 n^{2}(n-1)} \leqq \frac{32 n^{2}-32 n+10}{(2 n-1)(4 n-1)(4 n-3)},
$$

which is equivalent to

$$
8 n^{3}-24 n^{2}+13 n-3 \geqq 0 \text {. }
$$

We see easily that this cubic inequality in $n$ holds for $n \geqq 2.4$, which implies $n_{2}<2.4$. By numerical computation we have the following data:

$$
\begin{array}{ccc}
n & e_{n-1} & h(n) \\
2 & 2 & 35 / 18=1.94
\end{array}
$$

$2.1 \quad 2.036616 \cdots \quad 39.96 / 3 \times 2.1 \times 3.2=1.982142 \cdots$

$2.2 \quad 2.069615 \cdots \quad 45.24 / 3 \times 2.2 \times 3.4=2.016042 \cdots$

$2.3 \quad 2.099519 \cdots \quad 50.84 / 3 \times 2.3 \times 3.6=2.046698 \cdots$

$2.4 \quad 2.126750 \cdots \quad 56.76 / 3 \times 2.4 \times 3.8=2.074561 \cdots$

which show that $e_{n-1}>h(n)$ for $2 \leqq n \leqq 2.4$, and so $2.4<n_{1}$. Thus, we reach a contradiction and (4.1) has to hold.

Q.E.D.

LEMMA 4.2. $W(n, n)>0$ for $n>3 / 2$.

Proof. From (3.5) and (3.6) we have

$$
W(n, n)=-B W_{4}(n, n)=B n(n-1)^{3}(2 n-3)>0 \quad \text { for } n>3 / 2 .
$$

Q.E.D.

LEMMA 4.3. Near $x=1$, we have

$$
W(x, n)=\frac{B}{3} n\left(n^{2}-n+1\right)(x-1)^{4}+\cdots .
$$

Proof. Setting $x=1+y$, we obtain from (3.6) and (3.7)

$$
\begin{aligned}
& W_{4}(1+y, n)=120 n^{3}+20(n-1)^{2}(2 n-13) y \\
& \quad-4(n-1)\left(4 n^{2}+11 n-44\right) y^{2}+\left(16 n^{2}+6 n-37\right) y^{3}-(2 n+1) y^{4}, \\
& W_{5}(1+y, n)=120(n-1)^{3}+20(n-1)^{2}(8 n-19) y \\
& \quad+4(n-1)\left(21 n^{2}-101 n+109\right) y^{2}+3\left(8 n^{3}-54 n^{2}+112 n-71\right) y^{3} \\
& \quad+(n-2)\left(4 n^{2}-20 n+19\right) y^{4}-(n-1)(2 n-1) y^{5} .
\end{aligned}
$$

Hence, we obtain 


$$
\begin{aligned}
\frac{1}{B} W(1+y, n)= & -W_{4}(1+y, n)+\left(1-\frac{y}{n-1}\right)^{n-1} W_{5}(1+y, n) \\
= & -W_{4}(1+y)+\left\{1-y+\frac{n-2}{2(n-1)} y^{2}-\frac{(n-2)(n-3)}{6(n-1)^{2}} y^{3}\right. \\
& \left.+\frac{(n-2)(n-3)(n-4)}{24(n-1)^{3}} y^{4}+\cdots\right\} W_{5}(1+y) \\
= & \frac{n\left(n^{2}-n+1\right)}{3} y^{4}+\cdots,
\end{aligned}
$$

which implies this lemma.

Q.E.D.

LEMMA 4.4 . $W_{5}(x, n)>0$ for $0 \leqq x \leqq n$ with $n \geqq 2$.

Proof. Since we have (3.7) and (4.3)

$$
\left\{\begin{array}{l}
W_{5}(0)=12 n^{2}(2 n-1), \quad W_{5}(1)=120(n-1)^{3}, \\
W_{5}(n)=2 n^{7}-7 n^{6}+9 n^{5}-5 n^{4}+n^{3}=n^{3}(n-1)^{3}(2 n-1),
\end{array}\right.
$$

which are all positive for $n>1$. Therefore, we shall show that $W_{5}(x)>0$ for $0 \leqq x \leqq n$ with $n \geqq 2$. For simplicity, setting

$$
\left\{\begin{array}{l}
a_{0}=n^{2}(2 n-1), \quad a_{1}=n\left(16 n^{2}-36 n+9\right) \\
a_{2}=12 n^{3}-50 n^{2}+52 n-5=(2 n-5)\left(6 n^{2}-10 n+1\right) \\
a_{3}=8 n^{3}-70 n^{2}+130 n-71, \quad a_{4}=4 n^{3}-18 n^{2}+44 n-33 \\
a_{5}=(n-1)(2 n-1)=2 n^{2}-3 n+1
\end{array}\right.
$$

we have

$$
W_{5}(x, n)=12 a_{0}+3 a_{1} x+3 a_{2} x^{2}+a_{3} x^{3}+a_{4} x^{4}-a_{5} x^{5}
$$

First, we consider the quadratic polynomial of $x$ :

$$
\frac{1}{6} W_{5}^{(3)}(x)=a_{3}+4 a_{4} x-10 a_{5} x^{2},
$$

We have $a_{5}>0$ for $n>1$ and $a_{4}>0$ for $n \geqq 2$, and easily can show

$$
0<\frac{a_{4}}{5 a_{5}}<n \quad \text { for } n \geqq 2 \text {. }
$$

We have

$$
\begin{aligned}
D(n): & =(\text { its discriminant }) / 8=2 a_{4}^{2}+5 a_{3} a_{5} \\
& =32 n^{6}-208 n^{5}+532 n^{4}-1306 n^{3}+3238 n^{2}-4093 n+1823
\end{aligned}
$$


and $D^{\prime}(n)$ is $\searrow \nearrow$ in $2<n<\infty$, and $D^{\prime}(2)=-285, D^{\prime}(3)=-55$ and $D^{\prime}(3.1)=$ 647.65392. Hence $D(n)$ is $\searrow \nearrow$ in $2<n<\infty$. Since we have

$$
\begin{aligned}
& D(2)=45, \quad D(2.11)=3.8156 \cdots, \quad D(2.12)=-0.9263 \cdots, \\
& D(3.39)=-16.1886 \cdots, \quad D(3.40)=28.1905 \cdots,
\end{aligned}
$$

hence

$$
\begin{array}{ll}
D(n)>0 & \text { for } 2 \leqq n<2.11 \cdots \text { and } 3.39 \cdots<n<\infty, \\
D(n)<0 & \text { for } 2.11 \cdots<n<3.39 \cdots .
\end{array}
$$

We see that $a_{3}(n)$ is $\searrow \nearrow$ in $2<n<\infty$ and $a_{3}(2)=-27, a_{3}(6.4)=-9.048, a_{3}(6.5)$ $=13.5$ and hence

$$
\begin{aligned}
& a_{3}(n)<0 \quad \text { for } 2 \leqq n<6.4 \cdots \text { and } \\
& a_{3}(n)>0 \quad \text { for } n>6.4 \cdots \text { and } \\
& W_{5}^{(3)}(n)=-6\left(4 n^{4}+34 n^{3}-96 n^{2}+2 n+71\right)<0 \quad \text { for } n \geqq 2 .
\end{aligned}
$$

i) Case $2 \leqq n \leqq 2.11 \cdots$. From the above argument, the cubic polynomial of $x$ :

$$
\frac{1}{2} W_{5}^{\prime \prime}(x)=3 a_{2}+3 a_{3} x+6 a_{4} x^{2}-10 a_{5} x^{3}
$$

is $\searrow \nearrow$ in $0<x<n$. Since $a_{2}(n)<0$ for $2 \leqq n<2.5$, we consider its value at the root $\gamma$ of $W_{5}^{(3)}(x)=0$ :

$$
\gamma=\frac{2 a_{4}+\sqrt{2\left(2 a_{4} a_{4}+5 a_{3} a_{5}\right)}}{10 a_{5}}
$$

which becomes

$$
\frac{1}{5 a_{5}}\left[4 a_{4}^{3}+15 a_{3} a_{4} a_{5}+75 a_{2} a_{5}^{2}+\sqrt{2}\left(2 a_{4}^{2}+5 a_{3} a_{4}\right)^{3 / 2}\right],
$$

and, using that $a_{3}(n)$ is $\searrow$ in $a<n<4$, we can prove the quantity in the brackets is negative for $2 \leqq n \leqq 2.11 \cdots$. Thus, we see that $W_{5}{ }^{\prime \prime}(x)<0$ for $0 \leqq x \leqq n$ and so the graph of $W_{5}(x)$ is convex upward and so (4.4) implies $W_{5}(x)>0$ for $0 \leqq x \leqq n$.

ii) Case $2.11 \cdots<n<3.39 \cdots(D(n)<0) . \quad W_{5}^{(3)}(x)<0$ for $-\infty<x<+\infty$ and so $W_{5}^{\prime \prime}(x)$ is $\searrow$ in $0<x<n$. When $2.11 \cdots<n \leqq 2.5, W_{5}^{\prime \prime}(x)<0$ for $0<x \leqq n$ and so we have $W_{5}(x)>0$. When $2.5<n<3.39 \cdots, W_{5}^{\prime}(x)$ is $\nearrow$ or $\nearrow$ in $0<x<n$. Since we have

$$
\begin{aligned}
W_{5}^{\prime}(0) & =3 a_{1}>0 \quad \text { for } n \geqq 2, \\
W_{5}^{\prime}(n) & =6 n^{6}-33 n^{5}+33 n^{4}+6 n^{3}-9 n^{2}-3 n \\
& =3 n(n-1)^{2}\left(2 n^{3}-7 n^{2}-5 n-1\right)
\end{aligned}
$$


and $2 n^{3}-7 n^{2}-5 n-1$ is $\searrow \nearrow$ in $2<n<\infty,-23$ at $n=2,-1.1583 \cdots$ at $n=$ $4.13,0.2386 \cdots$ at $n=4.14$. Therefore $W_{5}^{\prime}(n)<0$ for $2 \leqq n<4.13 \cdots$. Hence we see that $W_{5}(x)$ is $\nearrow \backslash$ in $0<x<n$ with $2.5<n<3.39 \cdots$ and so (4.4) implies also $W_{5}(x)>0$ for $0 \leqq x \leqq n$.

iii) Case $3.39 \cdots \leqq n<\infty . \quad W_{5}^{\prime \prime}(x)$ is $\searrow \nearrow$ or $\nearrow \backslash$ in $0<x<n$ with $3.39 \cdots$ $\leqq n<6.4 \cdots$ or $6.4 \cdots \leqq n<\infty$ respectively. We have

and

$$
W_{5}^{\prime \prime}(0)=6 a_{2}>0 \quad \text { for } n>2.5 \text {, }
$$

$$
\begin{gathered}
W_{5}^{\prime \prime}(n)=2\left(4 n^{5}-54 n^{4}+80 n^{3}+42 n^{2}-57 n-15\right) \text { is } \searrow \nearrow \text { in } 2<n<\infty,<0 \\
\text { for } 2 \leqq n<11.7 \cdots \text { and }>0 \text { for } n>11.7 \cdots .
\end{gathered}
$$

Now, for the case $3.39 \cdots \leqq n \leqq 6.4 \cdots$, we consider the value of $W_{5}^{\prime \prime}(x)$ at the other root of $W_{5}^{(3)}(x)=0$ :

$$
\delta=\frac{2 a_{4}-\sqrt{2\left(2 a_{4}^{2}+5 a_{3} a_{5}\right)}}{10 a_{5}}
$$

which becomes

$$
\frac{1}{5 a_{5}}\left[4 a_{4}^{3}+15 a_{3} a_{4} a_{5}+75 a_{2} a_{3}^{2}-\sqrt{2}\left(2 a_{4}^{2}+5 a_{3} a_{4}\right)^{3 / 2}\right] .
$$

Since we have that

$$
\begin{aligned}
& 4 a_{4}^{3}+15 a_{3} a_{4} a_{5}+75 a_{2} a_{3}^{2} \\
= & 256 n^{9}-2496 n^{8}+13440 n^{7}-47976 n^{6}+111984 n^{5}-208542 n^{4} \\
& +368072 n^{3}-490365 n^{2}+364497 n-108978,
\end{aligned}
$$

which is $\nearrow$ in $3.3<n<\infty$, and $D(n)$ is $\nearrow$ in $3.1<n<\infty$, and computed the values of $4 a_{4}^{3}+15 a_{3} a_{4} a_{5}+75 a_{2} a_{3}^{2}$ and $\sqrt{2} D^{3 / 2}$ for $3.39 \leqq n \leqq 6.5$ with step $1 / 100$, we obtain

$$
4 a_{4}^{3}+15 a_{3} a_{4} a_{5}+75 a_{2} a_{3}^{2}-\sqrt{2} D^{3 / 2}>0 \quad \text { for } 3.39 \cdots \leqq n \leqq 6.5 \text {. }
$$

Therefore, $W_{5}^{\prime}(x)=3 a_{1}+6 a_{2} x+3 a_{3} x^{2}+4 a_{4} x^{3}-5 a_{5} x^{4}$ is $\nearrow$ in $0<x<n$. We have $a_{1}>0$ for $n \geqq 2$. Therefore $W_{5}(x)$ is $\nearrow \searrow$ or $\nearrow$ in $0<x<n$, which implies $W_{5}(x)$ $>0$ for $0 \leqq x \leqq n$ by (4.4).

Last, we consider the case $6.4 \cdots<n<\infty$. In this case $W_{5}^{\prime \prime}(x)$ is $\nearrow$ in $0<x<n$ and $W_{5}^{\prime \prime}(0)>0$. Hence $W_{5}^{\prime}(x)$ is $\nearrow \searrow$ or $\nearrow$ in $0<x<n$. We have $W_{5}^{\prime}(0)$ $>0$ and $W_{5}^{\prime}(n)>0$. Therefore $W_{5}^{\prime}(x)>0$ for $0 \leqq x \leqq n$, which implies $W_{5}(x)>0$ for $0 \leqq x \leqq n$ by (4.4). Thus, we have proved

$$
W_{5}(x, n)>0 \quad \text { for } 0 \leqq x \leqq n \text { with } n \geqq 2 .
$$

Q.E.D.

LEMMA 4.5. We have 


$$
\begin{aligned}
& W(x, n)=-B W_{4}(x, n)+(n-x)^{n-1} W_{5}(x, n)>0 \\
& \qquad \text { for } 0 \leqq x \leqq n, x \neq 1, \text { with } n \geqq 2 .
\end{aligned}
$$

Proof. If the above inequality does not hold, then there exist $\xi_{1}, \xi_{2}$ and $\eta_{1}, \eta_{2}$ such that

$$
\left\{\begin{array}{l}
0<\xi_{1} \leqq \xi_{2}<1 \quad \text { and } 1<\eta_{1} \leqq \eta_{2}<n \\
W\left(\xi_{i}\right)=W\left(\eta_{i}\right)=0, \quad i=1,2, \\
W^{\prime}\left(\xi_{1}\right) \leqq 0, \quad W^{\prime}\left(\xi_{2}\right) \geqq 0 \text { and } W^{\prime}\left(\eta_{1}\right) \leqq 0, \quad W^{\prime}\left(\eta_{2}\right) \geqq 0,
\end{array}\right.
$$

by Lemma 4.1-Lemma 4.3. By Lemma 4.4, the last conditions are equivalent to

$$
\begin{gathered}
(n-1) W_{4}(x) W_{5}(x)-(n-x)\left(W_{4}(x) W_{5}^{\prime}(x)-W_{4}^{\prime}(x) W_{5}(x)\right) \\
\geqq 0 \text { at } x=\xi_{1}, \quad \eta_{1} \text { and } \leqq 0 \text { at } x=\xi_{2}, \eta_{2} .
\end{gathered}
$$

Now, for simplicity, setting

$$
\left\{\begin{array}{l}
b_{0}=n(4 n-1)(4 n-3), \quad b_{1}=72 n^{3}-236 n^{2}+146 n-15, \\
b_{2}=16 n^{3}+76 n^{2}-190 n+71, \quad b_{3}=16 n^{2}+14 n-33, \\
b_{4}=2 n+1,
\end{array}\right.
$$

we have

$$
W_{4}(x, n)=4 b_{0}+b_{1} x-b_{2} x^{2}+b_{3} x^{3}-b_{4} x^{4} .
$$

From (4.5), (4.6) and (4.9), (4.10), we obtain

$$
\begin{aligned}
(n-1) & W_{4} W_{5}-(n-x)\left(W_{4} W_{5}^{\prime}-W_{4}^{\prime} W_{5}\right) \\
= & 12\left\{4(n-1) b_{0} a_{0}-n\left(b_{0} a_{1}-b_{1} a_{0}\right)\right\}+12\left\{n b_{0} a_{1}+(n-2) b_{1} a_{0}\right. \\
& \left.-2 n\left(b_{0} a_{2}+b_{2} a_{0}\right)\right\} x+3\left\{4(n+1) b_{0} a_{2}+(n-1) b_{1} a_{1}-4(n-3) b_{2} a_{0}\right. \\
& \left.-4 n b_{0} a_{3}-n\left(b_{1} a_{2}+b_{2} a_{1}\right)+12 n b_{3} a_{0}\right\} x^{2}+\left\{4(n+2) b_{0} a_{3}\right. \\
& +3 n b_{1} a_{2}-3(n-2) b_{2} a_{1}+12(n-4) b_{3} a_{0}-2 n\left(8 b_{0} a_{4}+b_{1} a_{3}-3 b_{3} a_{1}\right. \\
& \left.+24 b_{4} a_{0}\right\} x^{3}+\left\{4(n+3) b_{0} a_{4}+(n+1) b_{1} a_{3}-3(n-1) b_{2} a_{2}\right. \\
& +3(n-3) b_{3} a_{1}-12(n-5) b_{4} a_{0}+n\left(20 b_{0} a_{5}-3 b_{1} a_{4}+b_{2} a_{3}+3 b_{3} a_{2}\right. \\
& \left.\left.-9 b_{4} a_{1}\right)\right\} x^{4}+\left\{-4(n+4) b_{0} a_{5}+(n+2) b_{1} a_{4}-n b_{2} a_{3}+3(n-2) b_{3} a_{2}\right. \\
& \left.-3(n-4) b_{4} a_{1}+2 n\left(2 b_{1} a_{5}+b_{2} a_{4}-3 b_{4} a_{2}\right)\right\} x^{5}+\left\{-(n+3) b_{1} a_{5}\right. \\
& -(n+1) b_{2} a_{4}+(n-1) b_{3} a_{3}-3(n-3) b_{4} a_{2}-n\left(3 b_{2} a_{5}+b_{3} a_{4}\right. \\
& \left.\left.+b_{4} a_{3}\right)\right\} x^{6}+\left\{(n+2) b_{2} a_{5}+n b_{3} a_{4}-(n-2) b_{4} a_{3}+2 n b_{3} a_{5}\right\} x^{7} \\
& +\left\{-(n+1) b_{3} a_{5}-(n-1) b_{4} a_{4}-n b_{4} a_{5}\right\} x^{8}+n b_{4} a_{5} x^{9}
\end{aligned}
$$




$$
\begin{aligned}
= & n(n-1)\left[96 n^{3}\left(2 n^{2}-2 n+3\right)-96 n^{2}\left(6 n^{3}+5 n+8\right) x\right. \\
& +2 n\left(240 n^{4}+672 n^{3}+60 n^{2}+1140 n+351\right) x^{2} \\
& +\left(160 n^{5}-2080 n^{4}-400 n^{3}-2444 n^{2}-2388 n-225\right) x^{3} \\
& -2\left(240 n^{5}-480 n^{4}-500 n^{3}-608 n^{2}-1585 n-403\right) x^{4} \\
& +\left(288 n^{5}+192 n^{4}-608 n^{3}-260 n^{2}-2200 n-1135\right) x^{5} \\
& -\left(64 n^{5}+320 n^{4}-40 n^{3}+152 n^{2}-930 n-820\right) x^{6} \\
& +\left(96 n^{4}+80 n^{3}+140 n^{2}-212 n-335\right) x^{7} \\
& \left.-\left(40 n^{3}+16 n^{2}+2 n-70\right) x^{8}+\left(4 n^{2}-1\right) x^{9}\right] \\
= & n(n-1)(x-1)^{3} W_{6}(x, n),
\end{aligned}
$$

where

$$
\begin{aligned}
& W_{6}(x, n):=c_{6} x^{6}-c_{5} x^{5}+c_{4} x^{4}-c_{3} x^{3}+6 c_{2} x^{2}+192 c_{1} x-96 c_{0}, \\
& \left\{\begin{array}{l}
c_{6}=4 n^{2}-1, \quad c_{5}=40 n^{3}+4 n^{2}+2 n-67, \\
c_{4}=96 n^{4}-40 n^{3}+116 n^{2}-218 n-131, \\
c_{3}=64 n^{5}+32 n^{4}-40 n^{3}-212 n^{2}-282 n-225, \\
c_{2}=n\left(16 n^{4}-32 n^{3}-68 n^{2}+4 n-117\right), \\
c_{1}=n^{2}\left(3 n^{2}-2 n+4\right), \quad c_{0}=n^{3}\left(2 n^{2}-2 n+3\right) .
\end{array}\right.
\end{aligned}
$$

From the above argument, the last relations of (4.8) can be replaced by

$$
W_{6}\left(\xi_{1}\right) \leqq 0, \quad W_{6}\left(\xi_{2}\right) \geqq 0 \quad \text { and } \quad W_{6}\left(\eta_{1}\right) \geqq 0, \quad W_{6}\left(\eta_{2}\right) \leqq 0 .
$$

In the following, we shall prove the inequality:

$$
W_{6}(x, n)<0 \quad \text { for } 0 \leqq x \leqq n \text { with } n \geqq 2 .
$$

We see easily the following :

$$
\left\{\begin{array}{l}
W_{6}(0)=-96 c_{0}<0 \quad \text { for } n>0, \\
W_{6}(n)=-n^{3}\left(4 n^{5}-20 n^{4}+39 n^{3}-37 n^{2}+17 n-3\right)<0 \quad \text { for } n \geqq 2 .
\end{array}\right.
$$

First, we consider the quadratic polynomial of $x$ :

$$
\frac{1}{24} W_{6}^{(4)}(x)=15 c_{6} x^{2}-5 c_{5} x+c_{4} .
$$

We see easily that $c_{6}>0$ for $n>1 / 2, \quad c_{4}$ is $\nearrow$ in $2<n<\infty$, $\geqq 1113$, and $5 c_{5} /$ $\left(2 \times 15 c_{6}\right)=c_{5} / 6 c_{6}>n$ for $n \geqq 2$, and 


$$
\begin{aligned}
W_{6}^{(4)}(n) & =24\left(15 c_{6} n^{2}-5 c_{5} n+c_{4}\right) \\
& =-24\left(44 n^{4}+60 n^{3}-91 n^{2}-117 n+131\right)<0 \text { for } n \geqq 2 .
\end{aligned}
$$

Therefore, the cubic polynomial of $x$ :

$$
\frac{1}{6} W_{6}^{(3)}(x)=20 c_{6} x^{3}-10 c_{5} x^{2}+4 c_{4} x-c_{3}
$$

is $\nearrow$ in $0<x<n$. We see easily that $c_{3}$ is $\nearrow$ in $2<n<\infty, \geqq 603$, hence $W_{6}^{3}(0)$ $<0$, and

$$
\begin{aligned}
W_{6}^{(3)}(n) & =6\left(20 c_{6} n^{3}-10 c_{5} n^{2}+4 c_{4} n-c_{3}\right) \\
& =-6\left(232 n^{4}-464 n^{3}-10 n^{2}+242 n-225\right)<0 \text { for } n \geqq 2,
\end{aligned}
$$

since $232 n^{4}-464 n^{3}-10 n^{2}+242 n-225$ is $\nearrow$ in $2<n<\infty, \geqq 219$ for $n \geqq 2$. Then, we have

$$
\begin{aligned}
W_{6}^{(3)}\left(\frac{3 n}{4}\right) & =\frac{3}{8}\left(135 c_{6} n^{3}-90 c_{5} n^{2}+48 c_{4} n-16 c_{3}\right) \\
& =\frac{3}{8}\left(524 n^{5}-2792 n^{4}+5893 n^{3}-1042 n^{2}-1776 n+3600\right),
\end{aligned}
$$

which is $\nearrow$ in $2<n<\infty, \geqq(3 / 8) \times 15120=5670$ for $n \geqq 2$. Therefore, the polynomial of $x$ of 4 th order:

$$
\frac{1}{2} W_{6}^{\prime \prime}(x)=15 c_{6} x^{4}-10 c_{5} x^{3}+6 c_{4} x^{2}-3 c_{3} x+6 c_{2}
$$

is $\searrow \searrow$ in $0<x<n$. We can easily prove that

$$
\begin{aligned}
W_{6}{ }^{\prime \prime}(0) & =12 c_{2}<0 \quad \text { for } 2 \leqq n<3.40 \cdots \text { and } \\
& >0 \quad \text { for } 3.40 \cdots<n<\infty,
\end{aligned}
$$

since $16 n^{5}-32 n^{4}-68 n^{3}+4 n^{2}-117 n$ is $\searrow \nearrow$ in $2<n<\infty,-762$ at $n=2,-30.8393 \cdots$ at $3.4,1.6173 \cdots$ at 3.41 . Then, we have

and

$$
\begin{aligned}
W_{6}^{\prime \prime}(n) & =2\left(15 c_{6} n^{4}-10 c_{5} n^{3}+6 c_{4} n^{2}-3 c_{3} n+6 c_{2}\right) \\
& =2 n\left(44 n^{5}-280 n^{4}+589 n^{3}-410 n^{2}+84 n-27\right)>0 \text { for } n \geqq 2,
\end{aligned}
$$

$$
\begin{aligned}
W_{6}^{\prime \prime}(1) & =2\left(15 c_{6}-10 c_{5}+6 c_{4}-3 c_{3}+6 c_{2}\right) \\
& =-16\left(12 n^{5}-36 n^{4}+116 n^{3}-172 n^{2}+148 n-68\right)<0 \text { for } n \geqq 2 .
\end{aligned}
$$

Therefore, the polynomial of $x$ of 5 th order :

$$
W_{6}^{\prime}(x)=6 c_{6} x^{5}-5 c_{5} x^{4}+4 c_{4} x^{3}-3 c_{3} x^{2}+12 c_{2} x+192 c_{1}
$$

is $\searrow$ in $1<x<n$ with $n \geqq 2$, and $\searrow$ in $0<x<1$ when $2 \leqq n \leqq 3.40 \cdots$, and $\nearrow \searrow$ in $0<x<1$ when $3.40 \cdots<n<\infty$. We have 


$$
\begin{aligned}
W_{6}^{\prime}(0) & =192 c_{1}>0 \quad \text { for } n>0, \\
W_{6}^{\prime}(1) & =480\left(n^{4}-3 n^{2}+4 n^{2}-3 n+1\right) \\
& =480(n-1)^{2}\left(n^{2}-n+1\right)>0 \quad \text { for } n>1, \\
W_{6}^{\prime}(n) & =16 n^{7}-84 n^{6}+184 n^{5}-141 n^{4}-14 n^{3}+39 n^{2} \\
& =n^{2}(n-1)^{2}\left(16 n^{3}-52 n^{2}+64 n+39\right)>0 \text { for } n>1 .
\end{aligned}
$$

Thus, we see that $W_{6}^{\prime}(x)>0$ for $0 \leqq x \leqq 1$ with $n \geqq 2$, and hence $W_{6}(x)<0$ for $0 \leqq x \leqq 1$ with $n \geqq 2$ by (4.13).

Next, we consider the interval $1 \leqq x \leqq n . W_{6}^{\prime}(x)$ takes its minimum in $1 \leqq x$ $\leqq n$ at the root $\alpha$ of $W_{6}^{\prime \prime}(x)=0$. Since we have

$$
\begin{aligned}
\frac{1}{6} W_{6}^{(3)}\left(\frac{5 n}{6}\right)= & \frac{1}{(1.2)^{3}}\left(42.368 n^{5}-333.696 n^{4}+693.28 n^{3}\right. \\
& \left.-85.344 n^{2}-267.264 n+388.8\right)>0 \quad \text { for } n \geqq 2,
\end{aligned}
$$

we obtain

$$
W_{6}^{(3)}(x)>0 \quad \text { for } \frac{3}{4} n \leqq x \leqq \frac{5}{6} n
$$

from the facts stated above. Then, we have

$$
\begin{aligned}
\frac{1}{2} W_{6}{ }^{\prime \prime}\left(1+\frac{3}{10} n\right)= & -16.074 n^{6}+96.6 n^{5}-96.4215 n^{4}-390.25 n^{3}+809.06 n^{2} \\
& -868.1 n+544 \searrow \text { in } 2<n<\infty,<0 \quad \text { for } n \geqq 2,
\end{aligned}
$$

which implies

$$
1+\frac{3}{10} n<\alpha \quad \text { for } n \geqq 2 \text {. }
$$

We have also

which implies

$$
\begin{aligned}
& \frac{1}{2} W_{6}^{\prime \prime}\left(1+\frac{n}{3}\right)=-\frac{1}{27}\left(360 n^{6}-2792 n^{5}+3481 n^{4}+9218 n^{3}-24084 n^{2}\right. \\
& +22491 n-14688>0 \quad \text { for } 2 \leqq n<4.93 \cdots \\
& <0 \text { for } 4.93 \cdots<n<\infty \text {, }
\end{aligned}
$$

$$
\begin{array}{ll}
\alpha<1+\frac{n}{3} & \text { for } 2 \leqq n<4.93 \cdots \\
1+\frac{n}{3}<\alpha & \text { for } n>4.93 \cdots .
\end{array}
$$

We have $1+n / 3 \leqq 5 n / 6$ for $n \geqq 2$ and

$$
\begin{array}{r}
\frac{1}{6} W_{6}^{(3)}\left(1+\frac{3}{10} n\right)=17.36 n^{5}+82 n^{4}-335.14 n^{3}+497.3 n^{2}-383.2 n+351 \\
\text { is } \nearrow \text { in } 2<n<\infty, \geqq 760.2 \quad \text { for } n \geqq 2 .
\end{array}
$$


Hence we have

$$
W_{6}^{(3)}(x)>0 \quad \text { for } 1+\frac{3}{10} n \leqq x \leqq \frac{5}{6} n \text { with } n \geqq 2 .
$$

When $2 \leqq n<4.93 \cdots$, taking account of these facts we have

$$
\begin{aligned}
W_{6}^{\prime}(\alpha) \geqq & W_{6}^{\prime}\left(1+\frac{3}{10} n\right)+W_{6}^{\prime \prime}\left(1+\frac{3}{10} n\right) \times\left(\frac{1}{3} n-\frac{3}{10} n\right) \\
= & W_{6}^{\prime}\left(1+\frac{3}{10} n\right)+\frac{n}{30} W_{6}^{\prime \prime}\left(1+\frac{3}{10} n\right) \\
= & -\frac{1}{15}\left(143.1792 n^{7}-281.55 n^{6}-640.8648 n^{5}-1025.2475 n^{4}\right. \\
& \left.+10571.26 n^{3}-18697.45 n^{2}+16160 n-7200\right) \text { is } \nearrow \backslash \\
& \text { in } 2<n<\infty,>0 \quad \text { for } 2 \leqq n<2.62 \cdots \\
& \text { and }<0 \quad \text { for } 2.62 \cdots<n<4.93 \cdots,
\end{aligned}
$$

which implies

and so

$$
W_{6}^{\prime}(x)>0 \quad \text { for } 1 \leqq x \leqq n \text { with } 2 \leqq n<2.62 \cdots,
$$

$$
W_{6}(x)<0 \quad \text { for } 1 \leqq x \leqq n \text { with } 2 \leqq n<2.62 \cdots .
$$

Next, we have

$$
\begin{aligned}
W_{6}^{\prime}\left(1+\frac{3}{10} n\right)= & -\left(8.47368 n^{7}-12.33 n^{6}-49.15242 n^{5}-94.3665 n^{4}\right. \\
& \left.+758.688 n^{3}-1304.37 n^{2}+1113.6 n-480\right)
\end{aligned}
$$

which is $\searrow$ in $2.7<n<\infty,<0$ for $2.711 \cdots<n<\infty$. Therefore, for $n>2.711 \cdots$ we consider the root $\beta$ of $W_{6}^{\prime}(x)=0$ in $1<x<\alpha$ and the graph of $W_{6}(x)$ as shown in Fig. 2. The $x$-sections of the tangent lines at $\left(1, W_{6}(1)\right)$ and $\left(1+(3 / 10) n, W_{6}(1+(3 / 10) n)\right)$ are

$$
1-\frac{W_{6}(1)}{W_{6}^{\prime}(1)} \text { and } 1+\frac{3}{10} n-\frac{W_{6}\left(1+\frac{3}{10} n\right)}{W_{6}^{\prime}\left(1+\frac{3}{10} n\right)}
$$

respectively. The condition:

$$
-\frac{W_{6}(1)}{W_{6}^{\prime}(1)} \geqq \frac{3}{10} n-\frac{W_{6}\left(1+\frac{3}{10} n\right)}{W_{6}^{\prime}\left(1+\frac{3}{10} n\right)}
$$

is equivalent to

$$
W_{6}^{\prime}(1) W_{6}\left(1+\frac{3}{10} n\right) \leqq W_{6}^{\prime}\left(1+\frac{3}{10} n\right) \times\left\{W_{6}(1)+\frac{3}{10} n W_{6}^{\prime}(1)\right\}
$$




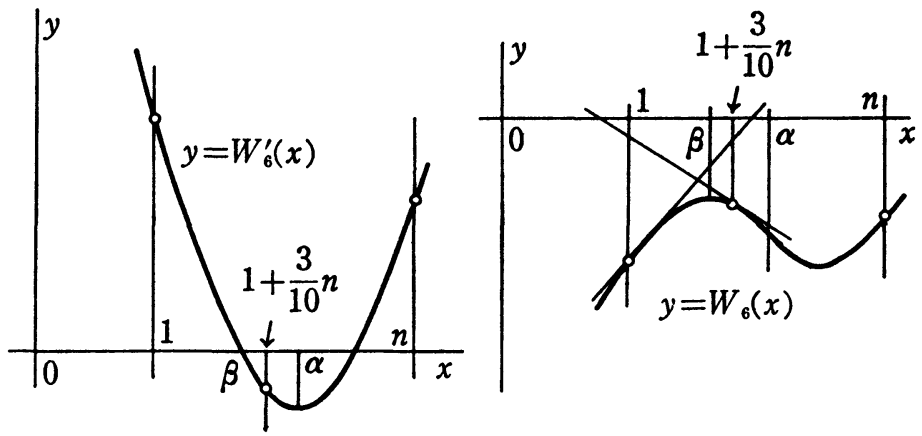

Fig. 2 .

Since we have

$$
W_{6}(1)=-160(n-1)^{3}\left(n^{2}-n+1\right), \quad W_{6}^{\prime}(1)=480(n-1)^{2}\left(n^{2}-n+1\right),
$$

the above condition becomes

$$
30 W_{6}\left(1+\frac{3}{10} n\right) \leqq(10-n) W_{6}^{\prime}\left(1+\frac{3}{10} n\right) .
$$

Computing carefully we obtain

$$
(10-n) W_{6}^{\prime}\left(1+\frac{3}{10} n\right)-30 W_{6}\left(1+\frac{3}{10} n\right)=n \times \Xi(n),
$$

where

$$
\begin{aligned}
\Xi(n)= & 39.8142 n^{7}-66.1248 n^{6}-469.99275 n^{5}+2853.7548 n^{4} \\
& -7804.674 n^{3}+10341.24 n^{2}-7957.5 n+3264,
\end{aligned}
$$

which is $\nearrow$ in $2.7<n<\infty, \geqq 3797.356219$ for $n \geqq 2.7$. Therefore, the above condition is satisfied for $n \geqq 2.7$. Hence, we obtain

$$
W_{6}(x)<0 \quad \text { for } 1<x<n \text { with } n>2.711 \cdots .
$$

Finally, on the rest interval $2.62 \cdots<n<2.711 \cdots$, we can show $W_{6}(x)<0$ for $1 \leqq x \leqq n$ by means of numerical computations by computors as before. Thus, we have proved

$$
W_{6}(x, n)<0 \quad \text { for } 0 \leqq x \leqq n \text { with } n \geqq 2,
$$

which contradicts to (4.12).

Q.E.D.

LEMMA 4.6. $W(x, n)$ is $\searrow$ in $0<x<1$ with $n \geqq 2$.

Proof. For simplicity, setting $n=m+1, x=1+y$, we obtain from (4.2) and (4.3) 


$$
\begin{aligned}
W_{4}(1+y)= & 120 m^{3}+20 m^{2}(2 m-11) y-4 m\left(4 m^{2}+19 m-29\right) y^{2} \\
& +\left(16 m^{2}+38 m-15\right) y^{3}-(2 m+3) y^{4}, \\
W_{5}(1+y)= & 120 m^{3}+20 m^{2}(8 m-11) y+4 m\left(21 m^{2}-59 m+29\right) y^{2} \\
& +3\left(8 m^{3}-30 m^{2}+28 m-5\right) y^{3}+\left(4 m^{3}-16 m^{2}+15 m-3\right) y^{4} \\
& -m(2 m+1) y^{5} .
\end{aligned}
$$

By Lemma 4.3, we have

and hence

$$
W(1)=W^{\prime}(1)=W^{\prime \prime}(1)=W^{(3)}(1)=0,
$$

$$
W^{\prime}(1+y)=\frac{1}{6} y^{3} W^{(4)}(1+\theta y), \quad 0<\theta<1 .
$$

From $W(1+y)=-B W_{4}(1+y)+(m-y)^{m} W_{5}(1+y)$, we obtain

$$
\begin{aligned}
\frac{1}{B} W^{(4)}(1+y)= & 24(2 m+3)+\left(1-\frac{y}{m}\right)^{m-4} \\
& \times\left\{\left(1-\frac{y}{m}\right)^{4} W_{5}^{(4)}-4\left(1-\frac{y}{m}\right)^{3} W_{5}^{(3)}+\frac{6(m-1)}{m}\left(1-\frac{y}{m}\right)^{2} W_{5}^{\prime \prime}\right. \\
& \left.-\frac{4(m-1)(m-2)}{m^{2}}\left(1-\frac{y}{m}\right) W_{5}^{\prime}+\frac{(m-1)(m-2)(m-3)}{m^{3}} W_{5}\right\} \\
= & 24(2 m+3)+\frac{1}{m^{4}}\left(1-\frac{y}{m}\right)^{m-4}\left\{8 m^{4}(m-2)(m+2)^{2}\right. \\
& -4 m^{3}\left(8 m^{4}+29 m^{3}+54 m^{2}+4 m-59\right) y \\
& +4 m^{2}(m+2)\left(21 m^{4}+43 m^{3}+68 m^{2}+68 m-38\right) y^{2} \\
& -m(m+2)(m+3)\left(40 m^{4}+114 m^{3}+110 m^{3}+123 m-33\right) y^{3} \\
& +(m+2)(m+3)(m+4)\left(4 m^{4}+28 m^{3}+19 m^{2}+12 m-3\right) y^{4} \\
& \left.-m(m+2)(m+3)(m+4)(m+5)(2 m+1) y^{5}\right\} .
\end{aligned}
$$

Since the polynomials of $m$ :

$$
\begin{aligned}
& 8 m^{4}+29 m^{3}+54 m^{2}+4 m-59,21 m^{4}+43 m^{3}+68 m^{2}+68 m-38, \\
& 40 m^{4}+114 m^{3}+110 m^{2}+123 m-33,4 m^{4}+28 m^{3}+19 m^{2}+12 m-3
\end{aligned}
$$

are all positive for $m \geqq 1$, we see easily that

$$
W^{(4)}(1+y)>0 \quad \text { for }-1 \leqq y<0 \text { with } m \geqq 2 .
$$

When $1 \leqq m<2$, we have 


$$
0<\left(1-\frac{y}{m}\right)^{m-4}<1 \quad \text { for } y<0,
$$

and hence for $-1<y<0$

Thus, we have proved

$$
\begin{aligned}
\frac{1}{B} W^{(4)}(1+y) & >24(2 m+3)+\frac{1}{m^{4}}\left(1-\frac{y}{m}\right)^{m-4} \\
& \times 8 m^{4}(m-2)(m+2)^{2} \\
> & 24(2 m+3)+8(m-2)(m+2)^{2} \\
& =8\left(m^{3}+2 m^{2}+2 m+1\right)>0 .
\end{aligned}
$$

$$
W^{\prime}(x, n)<0 \quad \text { for } 0 \leqq x<1 \text { with } n \geqq 2 .
$$

Q.E.D.

Proposition 3. $W(x, n)$ is positive for $0 \leqq x \leqq n, x \neq 1$ and decreasing in $0<x<1$ with $n \geqq 2$.

\section{$\S 5$. Some properties of $S(x, n)$.}

In this section, setting

$$
\left\{\begin{array}{l}
a_{0}=n(4 n-1)(4 n-3), \quad a_{1}=24 n^{3}-68 n^{2}+42 n-5, \\
a_{2}=8 n^{3}+20 n^{2}-51 n+20, \quad a_{3}=8 n^{2}-5
\end{array}\right.
$$

and

we have

$$
\left\{\begin{array}{l}
b_{0}=n^{2}(2 n-1), \quad b_{1}=n\left(16 n^{2}-32 n+9\right), \\
b_{2}=12 n^{3}-42 n^{2}+37 n-5, \quad b_{3}=8 n^{3}-52 n^{2}+87 n-40, \\
b_{4}=(n-1)\left(4 n^{2}-10 n+15\right)=4 n^{3}-14 n^{2}+15 n-5,
\end{array}\right.
$$

$$
\left\{\begin{array}{l}
S_{3}(x, n)=4 a_{0}+3 a_{1} x-2 a_{2} x^{2}+a_{3} x^{3}, \\
S_{4}(x, n)=12 b_{0}+3 b_{1} x+3 b_{2} x^{2}+b_{3} x^{3}+b_{4} x^{4}, \\
S(x, n)=-B S_{3}(x, n)+(n-x)^{n-1} S_{4}(x, n) .
\end{array}\right.
$$

LEMMA 5.1. $S(0, n)>0$ and $S(n, n)>0$ for $n \geqq 2$.

Proof. We have first

$$
\begin{aligned}
S(0, n) & =-4 B a_{0}+12 n^{n-1} b_{0} \\
& =-4 B n(4 n-1)(4 n-3)+12 n^{n+1}(2 n-1) \\
& =12 n^{2}(2 n-1) B\left\{e_{n-1}-\frac{(4 n-1)(4 n-3)}{3 n(2 n-1)}\right\}>0 \quad \text { for } n \geqq 2
\end{aligned}
$$


by (4.1). Next, we have

$$
\begin{aligned}
S(n, n) & =-B S_{3}(n, n)=B\left(8 n^{5}-32 n^{4}+43 n^{3}-22 n^{2}+3 n\right) \\
& =B n(n-1)^{2}\left(8 n^{2}-16 n+3\right)>0 \quad \text { for } n \geqq 2 .
\end{aligned}
$$

Q.E.D.

LEMMA 5.2. Near $x=1$, we have

$$
S(x, n)=\frac{B n(2 n-1)\left(n^{2}-n+5\right)}{6(n-1)}(x-1)^{4}+\cdots .
$$

Proof. Setting $x=1+y$, we obtain from (5.1)-(5.3)

Hence, we obtain

$$
\begin{aligned}
S_{3}(1+y, n)= & 60(n-1)^{2}(2 n-1)+10(n-1)\left(4 n^{2}-22 n+11\right) y \\
& -\left(16 n^{3}+16 n^{2}-102 n+55\right) y^{2}+\left(8 n^{2}-5\right) y^{3}, \\
S_{4}(1+y, n)= & 60(n-1)^{2}(2 n-1)+10(n-1)\left(16 n^{2}-40 n+17\right) y \\
& +3\left(28 n^{3}-122 n^{2}+154 n-55\right) y^{2} \\
& +3\left(8 n^{3}-36 n^{2}+49 n-20\right) y^{3} \\
& +(n-1)\left(4 n^{2}-10 n+5\right) y^{4} .
\end{aligned}
$$

$$
\begin{aligned}
\frac{1}{B} S(1+y, n)= & -S_{3}(1+y, n)+\left\{1-y+\frac{n-2}{2(n-1)} y^{2}-\frac{(n-2)(n-3)}{6(n-1)^{2}} y^{3}\right. \\
& \left.+\frac{(n-2)(n-3)(n-4)}{24(n-1)^{3}} y^{4}+\cdots\right\} \times S_{4}(1+y, n) \\
= & \frac{n(2 n-1)\left(n^{2}-n+5\right)}{6(n-1)} y^{4}+\cdots,
\end{aligned}
$$

which implies this lemma.

Q.E.D.

LEMMA 5.3. $S_{4}(x, n)>0$ for $0 \leqq x \leqq n$ with $n \geqq 2$.

Proof. From (5.2) and (5.5) we obtain

$$
\left\{\begin{aligned}
S_{4}(0) & =12 n^{2}(2 n-1)>0 \quad \text { for } n>1 / 2, \\
S_{4}(1) & =60(n-1)^{2}(2 n-1)>0 \quad \text { for } n>1, \\
S_{4}(n) & =4 n^{7}-6 n^{6}-n^{5}+4 n^{4}-n^{3} \\
& =n^{3}(n-1)^{2}\left(4 n^{2}+2 n-1\right)>0 \quad \text { for } n>1 .
\end{aligned}\right.
$$

We consider the quadratic polynomial of $x$ :

$$
\frac{1}{6} S_{4}^{\prime \prime}(x)=2 b_{4} x^{2}+b_{3} x+b_{2} .
$$


We have its discriminant

$$
\begin{aligned}
D(n) & =b_{3}^{2}-8 b_{2} b_{4} \\
& =-\left(320 n^{6}-1856 n^{5}+3232 n^{4}-136 n^{3}-5049 n^{2}+4880 n-1400\right)
\end{aligned}
$$

which is $\nearrow \backslash$ in $2<n<\infty, 124$ at $n=2$ and

$$
D(2.424)=0.5304 \cdots, \quad D(2.425)=-1.9072 \cdots,
$$

and hence $D(n)<0$ for $n>2.42 \cdots$. Therefore, we have

$$
S_{4}^{\prime \prime}(x)>0 \quad \text { for }-\infty<x<\infty \text { with } n>2.42 \cdots .
$$

Next, we see that

$$
-\frac{b_{3}}{4 b_{4}}=-\frac{8 n^{3}-52 n^{2}+87 n-40}{4(n-1)\left(4 n^{2}-10 n+5\right)} \text { is } \searrow \text { in } 2<n<\infty,
$$

because

$$
\left\{\frac{8 n^{3}-52 n^{2}+87 n-40}{(n-1)\left(4 n^{2}-10 n+5\right)}\right\}^{\prime}=\frac{3\left(32 n^{4}-152 n^{3}+266 n^{2}-200 n+55\right)}{(n-1)^{2}\left(4 n^{2}-10 n+5\right)^{2}}>0 \text { for } n \geqq 2 .
$$

We consider the condition:

which is equivalent to

$$
-\frac{b_{3}}{4 b_{4}}>n, \quad \text { i.e. } 4 n b_{4}+b_{3}<0,
$$

$$
16 n^{4}-48 n^{3}+8 n^{2}+67 n-40<0 .
$$

Since the left hand side is $\nearrow$ in $2<n<\infty$, we see that this condition is equivalent to $2 \leqq n<2.04 \cdots$. Then, we consider the condition :

which is equivalent to

$$
-\frac{b_{3}}{4 b_{4}}<1, \quad \text { i. e. } 4 b_{4}+b_{3}>0
$$

$$
24 n^{3}-108 n^{2}+147 n-60>0 .
$$

Since the left hand side is $\nearrow$ in $2<n<\infty$, we see that this condition is equivalent to $n>2.33 \cdots$.

On the other hand, we have

$$
\begin{aligned}
S_{4}{ }^{\prime \prime}(0)=6 b_{2} & =6\left(12 n^{3}-42 n^{2}+37 n-5\right) \nearrow \text { in } 2<n<\infty, \\
& <0 \quad \text { for } 2 \leqq n<2.16 \cdots \text { and } \\
& >0 \quad \text { for } n>2.16 \cdots
\end{aligned}
$$

and

$$
\begin{aligned}
S_{4}{ }^{\prime \prime}(n) & =6\left(8 n^{5}-20 n^{4}-10 n^{3}+35 n^{2}-3 n-5\right) \nearrow \text { in } 2<n<\infty, \\
& <0 \quad \text { for } 2 \leqq n<2.24 \cdots \text { and } \\
& >0 \quad \text { for } n>2.24 \cdots .
\end{aligned}
$$


Taking account of these facts, we consider our subject in the following cases.

i) Case $2 \leqq n \leqq 2.16 \cdots$.

We see easily $S_{4}^{\prime \prime}(x)<0$ for $0 \leqq x \leqq n$. Therefore the graph of $S_{4}(x)$ is convex upward and so (5.6) implies

$$
S_{4}(x)>0 \quad \text { for } 0 \leqq x \leqq n .
$$

ii) Case $2.16 \cdots<n \leqq 2.24 \cdots$.

We have

$$
S_{4}^{\prime}(x)=3 b_{1}+6 b_{2} x+3 b_{3} x^{2}+4 b_{4} x^{3} \text { is } \nearrow \text { in } 0<x<n .
$$

$$
\begin{aligned}
S_{4}^{\prime}(0) & =3 b_{1}=3 n\left(16 n^{2}-32 n+9\right)>0 \quad \text { for } n \geqq 2, \\
S_{4}^{\prime}(n) & =16 n^{6}-32 n^{5}-24 n^{4}+37 n^{3}+6 n^{2}-3 n \\
& =n(n-1)\left(16 n^{4}-16 n^{3}-40 n^{2}-3 n+3\right),
\end{aligned}
$$

and $16 n^{4}-16 n^{3}-40 n^{2}-3 n+3$ is $\nearrow$ in $2<n<\infty$,

$$
\begin{array}{ll}
<0 & \text { for } 2<n<2.17 \cdots \text { and } \\
>0 & \text { for } n>2.17 \cdots .
\end{array}
$$

Therefore $S_{4}(x)$ is $\nearrow \searrow$ or $\nearrow$ in $0<x<n$. Then, (5.6) also implies $S_{4}(x)>0$ for $0 \leqq x \leqq n$.

iii) Case $2.42 \cdots<n<\infty$ (i. e. $D(n)<0$ ).

$S_{4}^{\prime}(x)$ is $\nearrow$ in $-\infty<n<\infty$ and so $S_{4}^{\prime}(x)>0$ for $x \geqq 0$, hence $S_{4}(x)$ is $\nearrow$ in $0<x<n$, and $S_{4}(x)>0$ for $0 \leqq x \leqq n$ by (5.6).

iv) Case $2.24 \cdots<n<2.42 \cdots$.

$$
S_{4}^{\prime}(x) \text { is } \nearrow \searrow \nearrow \text { in } 0<x<n .
$$

Let $\alpha$ be the root of $S_{4}^{\prime \prime}(x)=0$ :

$$
\alpha(n)=\frac{-b_{3}+\sqrt{b_{3} b_{3}-8 b_{2} b_{4}}}{4 b_{4}} .
$$

In this range of $n$, evaluating $\alpha(n)$ by computor, we presumed it as

and we have

$$
17.9-7 n<\alpha(n)<18.1-7 n \quad \text { for } 2.2 \leqq n<2.42 \cdots,
$$

$$
\begin{gathered}
\frac{1}{6} S_{4}{ }^{\prime \prime}(17.9-7 n)=392 n^{5}-3432.8 n^{4}+11569.28 n^{3} \\
-18561.28 n^{2}+13992.6 n-3925.1 \text { is } \nearrow \searrow \nearrow \\
\text { in } 2.2<n<2.4 \text { and } \nearrow \text { in } 2.4<n<\infty, \\
<0 \quad \text { for } 2.2 \leqq n<2.422 \cdots
\end{gathered}
$$

and 
Hence we obtain

$$
\begin{gathered}
\frac{1}{6} S_{4}{ }^{\prime \prime}(18.1-7 n)=392 n^{5}-3455.2 n^{4}+11706.88 n^{3} \\
-18857.28 n^{2}+14254 n-4005.1 \text { is } \nearrow \searrow \nearrow \\
\text { in } 2.2<n<2.4 \text { and } \nearrow \text { in } 2.4<n<\infty, \\
>0 \quad \text { for } n \geqq 2.2 .
\end{gathered}
$$

$$
\begin{cases}17.9-7 n<\alpha(n) & \text { for } 2.2 \leqq n<2.422 \cdots, \\ \alpha(n)<18.1-7 n & \text { for } 2.2 \leqq n<2.42 \cdots\end{cases}
$$

(here, $2.42 \cdots$ means the root of $D(n)=0$ ). Regarding the symmetric axis of the quadratic polynomial $2 b_{4} x^{2}+b_{3} x+b_{2}$, the condition:

$$
-\frac{b_{3}}{4 b_{4}}=-\frac{8 n^{3}-52 n^{2}+87 n-40}{4(n-1)\left(4 n^{2}-10 n+5\right)}<17.9-7 n
$$

is equivalent to

$$
112 n^{4}-686.4 n^{3}+1474.7 n^{2}-1301 n+398<0 .
$$

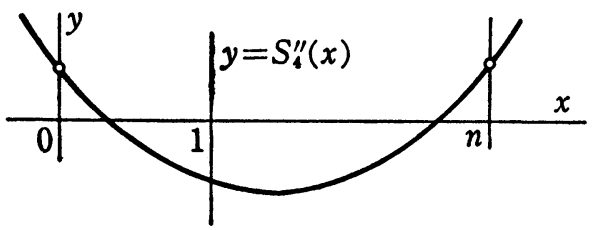

The left hand side is $\searrow \nearrow$ in $2.2<n<2.4$ and $\nearrow$ in $2.4<n<\infty$, and negative for $2.2 \leqq n \leqq 2.43$. Hence we see that

$$
-\frac{b_{3}}{4 b_{4}}<17.9-7 n \quad \text { for } 2.2 \leqq n \leqq 2.43 .
$$

Since we have

$$
\begin{aligned}
S_{4}^{\prime}(17.9-7 n)=- & 5488 n^{6}+62484.8 n^{5}-289752.96 n^{4} \\
& +695978.824 n^{3}-904952.944 n^{2}+596602.35 n-153692.98,
\end{aligned}
$$

taking account of (5.8) and (5.9), we obtain

$$
\begin{aligned}
S_{4}^{\prime}(\alpha)> & S_{4}^{\prime}(17.9-7 n)+0.2 \times S_{4}^{\prime \prime}(17.9-7 n) \\
= & -5488 n^{6}+62955.2 n^{5}-293872.32 n^{4}+709861.96 n^{3} \\
& -927226.48 n^{2}+613393.47 n-158403.1:=\mu(n) .
\end{aligned}
$$

Since $\mu^{(4)}(n)<0$ for $2.24<n<\infty$, and $\mu^{\prime \prime}(n)<0$ for $2.24<n<\infty$, hence $\mu(n)$ is $\nearrow$ in $2.24<n<\infty$. Since $\mu(2.24)=50.32 \cdots$ and $\mu(2.42)=197.31 \cdots$, and so $\mu(n)$ $>0$ for $2.24 \leqq n \leqq 2.42$, which implies $S_{4}^{\prime}(\alpha)>0$. Since $S_{4}^{\prime}(0)=3 b_{1}>0$, we have $S_{4}^{\prime}(x)>0$ for $0 \leqq x \leqq n$. Hence, we obtain also 


$$
S_{4}(x)>0 \quad \text { for } 0 \leqq x \leqq n .
$$

Regarding the rest very narrow range $2.42<n<2.42 \cdots$ (the root of $D(n)=0$ ), we can drow the same result by numerical treatment by computor. Thus, we have proved

$$
S_{4}(x, n)>0 \quad \text { for } 0 \leqq x \leqq n \text { with } n \geqq 2 \text {. }
$$

Q.E.D.

Proposition 4. We have

$$
\begin{gathered}
S(x, n)=-B S_{3}(x, n)+(n-x)^{n-1} S_{4}(x, n)>0 \\
\text { for } 0 \leqq x \leqq n, x \neq 1 \text { with } n \geqq 2 .
\end{gathered}
$$

Proof. If the above inequality does not hold, then there exist $\xi_{1}, \xi_{2}$ and $\eta_{1}$, $\eta_{2}$ such that

$$
\left\{\begin{array}{l}
0<\xi_{1} \leqq \xi_{2}<1 \quad \text { and } 1<\eta_{1} \leqq \eta_{2}<n \\
S\left(\xi_{i}\right)=S\left(\eta_{i}\right)=0, \quad i=1,2, \\
S^{\prime}\left(\xi_{1}\right) \leqq 0, \quad S^{\prime}\left(\xi_{2}\right) \geqq 0 \quad \text { and } S_{i}^{\prime}\left(\eta_{1}\right) \leqq 0, \quad S^{\prime}\left(\eta_{2}\right) \geqq 0
\end{array}\right.
$$

by Lemma 5.1 and Lemma 5.2. By Lemma 5.3, the last ${ }_{\mathbf{2}}^{7}$ conditions are equivalent to

$$
\begin{gathered}
(n-1) S_{3}(x) S_{4}(x)-(n-x)\left(S_{3}(x) S_{4}^{\prime}(x)-S_{3}^{\prime}(x) S_{4}(x)\right) \\
\geqq 0 \text { at } x=\xi_{1}, \eta_{1} \text { and } \leqq 0 \text { at } x=\xi_{2}, \eta_{2} .
\end{gathered}
$$

By (5.1)-(5.3), working out the computation very carefully, we obtain

$$
\begin{aligned}
&(n-1) S_{3} S_{4}-(n-x)\left(S_{3} S_{4}^{\prime}-S_{3}^{\prime} S_{4}\right) \\
&=n(n-1)\left[n^{3}\left(192 n^{2}-192 n+192\right)\right. \\
& \quad-n^{2}\left(576 n^{3}-192 n^{2}-288 n+624\right) x \\
& \quad+n\left(480 n^{4}+768 n^{3}-1680 n^{2}+720 n+660\right) x^{2} \\
& \quad+\left(160 n^{5}-1600 n^{4}+1240 n^{3}+1540 n^{2}-1550 n-225\right) x^{3} \\
& \quad-\left(480 n^{5}-1120 n^{4}-680 n^{3}+2720 n^{2}-640 n-700\right) x^{4} \\
& \quad+\left(288 n^{5}-288 n^{4}-1032 n^{3}+1080 n^{2}+780 n-750\right) x^{5} \\
&-\left(64 n^{5}+32 n^{4}-392 n^{3}+16 n^{2}+580 n-300\right) x^{6} \\
&\left.+\left(32 n^{4}-80 n^{3}+20 n^{2}+50 n-25\right) x^{7}\right] \\
&= n(n-1)(x-1)^{3} T_{4}(x, n),
\end{aligned}
$$

where

$$
T_{4}(x, n)=c_{4} x^{4}-c_{3} x^{3}+12 c_{2} x^{2}+48 c_{1} x-192 c_{0},
$$




$$
\left\{\begin{array}{l}
c_{4}=32 n^{4}-80 n^{3}+20 n^{2}+50 n-25 \\
c_{3}=64 n^{5}-64 n^{4}-152 n^{3}-44 n^{2}+430 n-225 \\
c_{2}=n\left(8 n^{4}-16 n^{3}-28 n^{2}+96 n-55\right) \\
c_{1}=n^{2}\left(8 n^{2}-18 n+13\right), \quad c_{0}=n^{3}\left(n^{2}-n+1\right)
\end{array}\right.
$$

From the above relation, the last relations of (5.10) are equivalent to

$$
T_{4}\left(\xi_{1}\right) \leqq 0, \quad T_{4}\left(\xi_{2}\right) \geqq 0 \quad \text { and } \quad T_{4}\left(\eta_{1}\right) \geqq 0, \quad T_{4}\left(\eta_{2}\right) \leqq 0 .
$$

In the following, we shall prove the inequality:

$$
T_{4}(x, n)<0 \quad \text { for } 0 \leqq x \leqq n \text { with } n \geqq 2 .
$$

We see easily the following:

$$
\left\{\begin{aligned}
T_{4}(0) & =-192 c_{0}<0 \quad \text { for } n>0, \\
T_{4}(1) & =-40\left(4 n^{5}-12 n^{4}+33 n^{3}-46 n^{2}+26 n-5\right) \\
& =-40(n-1)(2 n-1)\left(2 n^{3}-3 n^{2}+11 n-5\right)<0 \text { for } n>1, \\
T_{4}(n) & =-n^{3}(n-1)\left(32 n^{4}-48 n^{3}-28 n^{2}+22 n-3\right)<0 \text { for } n \geqq 2,
\end{aligned}\right.
$$

and $c_{4}=32 n^{4}-80 n^{3}+20 n^{2}+50 n-25$ is $\nearrow$ in $2<n<\infty$, $\geqq 27$ for $n \geqq 2$. Now, we consider the quadratic polynomial of $x$ :

$$
\frac{1}{6} T_{4}^{\prime \prime}(x)=2 c_{4} x^{2}-c_{3} x+4 c_{2} \text {. }
$$

Since we have

$$
\begin{gathered}
c_{2}=n\left(8 n^{4}-16 n^{3}-28 n^{2}+96 n-55\right) \nearrow \text { in } 2<n<\infty, \\
\geqq 50 \quad \text { for } n \geqq 2, \\
\frac{1}{6} T_{4}^{\prime \prime}(1)=-32 n^{5}+64 n^{4}-120 n^{3}+468 n^{2}-550 n+175 \\
\searrow \text { in } 2<n<\infty, \leqq-13 \quad \text { for } n \geqq 2, \\
\frac{1}{6} T_{4}{ }^{\prime \prime}(n)=-n\left(64 n^{4}-128 n^{3}-32 n^{2}+96 n-5\right) \\
\searrow \text { in } 2<n<\infty, \leqq-118 \quad \text { for } n \geqq 2,
\end{gathered}
$$

we see that $T_{4}^{\prime \prime}(x)<0$ for $1 \leqq x \leqq n$ and

$$
\begin{aligned}
& T_{4}^{\prime}(x)=4 c_{4} x^{3}-3 c_{3} x^{2}+24 c_{2} x+48 c_{1} \\
& \nearrow \searrow \text { in } 0<x<1 \\
& \text { and } \searrow \text { in } 1<x<n \text { with } n \geqq 2 .
\end{aligned}
$$

Then, we have 


$$
\left\{\begin{array}{c}
T_{4}^{\prime}(0)=48 c_{1}=48 n^{2}\left(8 n^{2}-18 n+13\right)>0 \quad \text { for } n \geqq 2, \\
T_{4}^{\prime}(1)=5\left(64 n^{4}-280 n^{3}+628 n^{2}-482 n+115\right) \\
\nearrow \text { in } 2<n<\infty, \geqq 2235 \quad \text { for } n \geqq 2, \\
T_{4}^{\prime}(n)=-n^{2}\left(64 n^{5}-64 n^{4}-152 n^{3}-44 n^{2}-50 n+21\right) \\
\searrow \text { in } 2<n<\infty,>0 \text { for } 2 \leqq n<2.24 \cdots \\
\text { and }<0 \quad \text { for } n>2.24 \cdots .
\end{array}\right.
$$

Therefore, we have

and hence

$$
T_{4}^{\prime}(x)>0 \quad \text { for } 0 \leqq x \leqq 1 \text { with } n \geqq 2,
$$

$$
T_{4}(x)<0 \quad \text { for } 0 \leqq x \leqq 1 \text { with } n \geqq 2
$$

by (5.14). When $2 \leqq n \leqq 2.24 \cdots$, we see that

$$
T_{4}^{\prime}(x)>0 \quad \text { for } 1<x<n
$$

and $T_{4}(x)$ is $\nearrow$ in $1<x<n$. Thus we obtain

$$
T_{4}(x)<0 \quad \text { for } 1 \leqq x \leqq n .
$$

When $2.24 \cdots<n<\infty$, we see that

$$
T_{4}(x) \nearrow \searrow \text { in } 1<x<n \text { and convex upward. }
$$

Let $\alpha_{1}$ and $\alpha_{n}$ be the $x$-coordinate sections of the tangent lines of the graph of $T_{4}(x)$ at $\left(1, T_{4}(1)\right)$ and $\left(n, T_{4}(n)\right)$, respectively. Since

$$
\alpha_{1}=1-\frac{T_{4}(1)}{T_{4}^{\prime}(1)}, \quad \alpha_{n}=n-\frac{T_{4}(n)}{T_{4}^{\prime}(n)},
$$

we have

$$
\begin{aligned}
\alpha_{1}-\alpha_{n}= & \frac{T_{4}(n)}{T_{4}^{\prime}(n)}-\frac{T_{4}(1)}{T_{4}^{\prime}(1)}-(n-1) \\
= & \frac{n(n-1)\left(32 n^{4}-48 n^{3}-28 n^{2}+22 n-3\right)}{64 n^{5}-64 n^{4}-152 n^{3}-44 n^{2}-50 n+21} \\
& +\frac{8(n-1)\left(4 n^{4}-8 n^{3}+25 n^{2}-21 n+5\right)}{64 n^{4}-280 n^{3}+628 n^{2}-482 n+115}-(n-1) \\
= & \frac{n-1}{2}\left[-\frac{32 n^{4}-96 n^{3}-88 n^{2}-44 n+21}{64 n^{5}-64 n^{4}-152 n^{3}-44 n^{2}-50 n+21}\right. \\
& \left.+\frac{152 n^{3}-228 n^{2}+146 n-35}{64 n^{4}-280 n^{3}+628 n^{2}-482 n+115}\right] .
\end{aligned}
$$

Since the denominators of the both fractional expressions in the brackets are 
positive for $n>2.24 \cdots$, the condition : $\alpha_{1} \geqq \alpha_{n}$ is equivalent to

$$
\begin{aligned}
& \left(64 n^{5}-64 n^{4}-152 n^{3}-44 n^{2}-50 n+21\right)\left(152 n^{3}-228 n^{2}+146 n-35\right) \\
& \quad-\left(64 n^{4}-280 n^{3}+628 n^{2}-482 n+115\right)\left(32 n^{4}-96 n^{3}-88 n^{2}-44 n+21\right) \geqq 0,
\end{aligned}
$$

i.e.

$$
\begin{gathered}
1280 n^{8}-1536 n^{7}-6752 n^{6}+11712 n^{5}-4312 n^{4} \\
+2604 n^{3}-5804 n^{2}+3333 n-525 \geqq 0,
\end{gathered}
$$

whose left hand side is $\nearrow$ in $2<n<\infty, 8493$ at $n=2$. Hence, we see that $\alpha_{1}>\alpha_{n}$ for $n>2.24 \cdots$, which implies

$$
T_{4}(x)<0 \quad \text { for } 1 \leqq x \leqq n .
$$

thus, we have proved $T_{4}(x)<0$ for $0 \leqq x \leqq n$ with $n \geqq 2$. Therefore (5.13) contradicts this fact, which implies this proposition.

Q.E.D.

The following proposition will be proved also in [24].

Proposition 5. $S(x, n)$ is decreasing in $0<x<1$ with $n \geqq 2$ and increasing in $1<x<n$ with $2 \leqq n \leqq(11+\sqrt{77}) / 4=4.9437410 \cdots$.

\section{§6. Proof of Main Theorem.}

All the facts expected to obtain $\partial V\left(x, x_{1}\right) / \partial x>0$ described at the end of $\S 3$ are satisfied by Propositions 3,4 and 5 for $2 \leqq n \leqq(11+\sqrt{77}) / 4$.

Regarding $\Psi(x, n)$ defined by (3.13), we have

$$
\begin{aligned}
\Psi(x, n)= & \frac{x \sqrt{n-x}}{(n-1)(1-x)^{5}}\left[-\frac{(n-1) x}{(1-x)^{2}}\{\tilde{\lambda}(X)-\tilde{\lambda}(x)\} W(x, n)\right. \\
& +S(x, n)+\left(\frac{1-x}{X-1}\right)^{5} \frac{X \sqrt{n-X}}{x \sqrt{n-x}} S(X, n) \\
= & \frac{x \sqrt{n-x}}{(n-1)(1-x)^{5}} \Phi(x, n),
\end{aligned}
$$

where $X=X_{n}(x)$ and

$$
\begin{aligned}
\Phi(x, n)= & -\frac{(n-1) x}{(1-x)^{2}}\{\tilde{\lambda}(X)-\tilde{\lambda}(x)\} W(x, n) \\
& +S(x, n)+\left(\frac{1-x}{X-1}\right)^{5}\left(\frac{n-x}{n-X}\right)^{n-3 / 2} S(X, n) .
\end{aligned}
$$

In the following, we shall prove $\Phi(x, n)>0$ for $0<x<1$ with $2 \leqq n<$ $(11+\sqrt{77}) / 4$ by means of numerical evaluation by computors partially, which implies our Main Theorem. 
LEMMA 6.1. We have

$$
\begin{aligned}
& \text { (i) } \lim _{x \rightarrow 0} \Phi(x, n)=\infty, \\
& \text { (ii) } \Phi(x, n) \text { is } \searrow 0 \text { near } x=1 .
\end{aligned}
$$

Proof. (i) Since we have

$$
\begin{aligned}
\lim _{x \rightarrow 0} x\{\tilde{\lambda}(X)-\tilde{\lambda}(x)\} & =\lim _{x \rightarrow 0} \frac{\tilde{\lambda}(X)-\tilde{\lambda}(x)}{1 / x} \\
& =\lim _{x \rightarrow 0} \frac{1}{-1 / x^{2}} \frac{n(1-x)}{(n-1) x(n-x)}\left(\frac{1}{X}-\frac{1}{x}\right) \\
& =\lim _{x \rightarrow 0} \frac{n(1-x)}{(n-1)(n-x)}\left(1-\frac{x}{X}\right)=\frac{1}{n-1}
\end{aligned}
$$

by Lemma 4.1 in (IX), we obtain

$$
\lim _{x \rightarrow 0} \Phi(x, n)=-W(0, n)+S(0, n)+S(n, n) \lim _{x \rightarrow 0}\left(\frac{n-x}{n-X}\right)^{n-3 / 2}=\infty .
$$

(ii) By Lemma 7.1 in (III) we have

$$
\tilde{\lambda}(x)=\tilde{\lambda}(1)-\frac{2 n-1}{2(n-1)^{2}}(x-1)^{2}+\frac{3 n^{2}-6 n+2}{3(n-1)^{3}}(x-1)^{3}+\cdots .
$$

Setting $x=1-t$ and $X=1+s$, we obtain

$$
\begin{aligned}
\tilde{\lambda}(X)-\tilde{\lambda}(x) & =-\frac{2 n-1}{2(n-1)^{2}}\left(s^{2}-t^{2}\right)+\frac{3 n^{2}-6 n+2}{3(n-1)^{3}}\left(s^{3}+t^{3}\right)+\cdots \\
& =-\frac{2 n-1}{2(n-1)^{2}}\left(\frac{4(n-2)}{3(n-1)} t^{3}+\cdots\right)+\frac{3 n^{2}-6 n+2}{3(n-1)^{3}}\left(2 t^{3}+\cdots\right)+\cdots \\
& =\frac{2 n}{3(n-1)^{2}} t^{3}+\cdots
\end{aligned}
$$

by Lemma 3.1. Therefore, by Lemma 4.3 and Lemma 5.2 we obtain

$$
\begin{aligned}
\Phi(x, n)= & -(n-1) \cdot \frac{2 n}{3(n-1)^{2}} \cdot \frac{B}{3} n\left(n^{2}-n+1\right) t^{5}+\cdots \\
& +\left(\frac{B n(2 n-1)\left(n^{2}-n+5\right)}{6(n-1)} t^{4}+\cdots\right) \\
& +\left(\frac{B n(2 n-1)\left(n^{2}-n+5\right)}{6(n-1)} s^{4}+\cdots\right),
\end{aligned}
$$

which implies (ii).

Q.E.D.

Since we have $\lim _{x \rightarrow 0} \tilde{\lambda}(X)-\tilde{\lambda}(x)=\infty$ by Lemma 4.1 in (IX), we arrange the following lemma to make easy evaluations at the both ends of $0 \leqq x \leqq 1$. 
LEMMA 6.2. We have

$$
x^{2}\{\tilde{\lambda}(X)-\tilde{\lambda}(x)\}<\frac{k_{n}}{n-1} x(1-x)^{3} \quad \text { for } 0<x<1,
$$

where $X=X_{n}(x), k_{n}=n / 2$ for $n \geqq 3$ and $=n^{2} / 3(n-1)$ for $2 \leqq n<3$.

Proof. Considering the following expression:

$$
\frac{x^{2}\{\tilde{\lambda}(X)-\tilde{\lambda}(x)\}}{x(1-x)^{3}}=\frac{\tilde{\lambda}(X)-\tilde{\lambda}(x)}{\frac{1}{x}(1-x)^{3}},
$$

and noticing Cauchy's mean value theorem and a formula in the proof of Lemma 4.1 in (IX) we consider

$$
\frac{\frac{n}{(n-1) x(n-x)}\left(\frac{1}{X}-\frac{1}{x}\right)}{-\frac{1}{x^{2}}+3-2 x}=\frac{n}{n-1} \cdot \frac{1}{(n-x)(2 x+1) X} \cdot \frac{X-x}{1-x} .
$$

By Proposition 4 in (IV), $(X-1) /(1-x)$ is $\searrow 1$ as $x \rightarrow 1$, therefore the above expression

$$
<\frac{n^{2}}{n-1} \cdot \frac{1}{(n-x)(2 x+1) X}<\frac{n^{2}}{n-1} \cdot \frac{1}{(n-x)(2 x+1)(2-x)} .
$$

On the other hand, the cubic polynomial of $x$ :

$$
(n-x)(2 x+1)(2-x)=2 n+(3 n-2) x-(2 n+3) x^{2}+2 x^{3}
$$

is $\nearrow$ in $0<x<1$ and equal to $2 n$ at $x=0$ and $3(n-1)$ at $x=1$. Therefore, we obtain

$$
\frac{x^{2}\{\tilde{\lambda}(X)-\tilde{\lambda}(x)\}}{x(1-x)^{3}}<\frac{k_{n}}{n-1} \quad \text { for } 0<x<1
$$

Q.E.D.

Remark. Regarding the expressions in $\Phi(x, n)$, we have the following facts.

(i) $\frac{x}{(1-x)^{2}}$ is $\nearrow$ in $0<x<1$;

(ii) $\tilde{\lambda}(X)-\tilde{\lambda}(x)$ is $\searrow$ in $0<x<1$, from $\infty$ to 0

(Lemma 4.1 in (IX));

(iii) $\frac{1-x}{X-1}$ is $\nearrow$ in $0<x<1$, from $\frac{1}{n-1}$ to 1 ;

(iv) $\left(\frac{n-x}{n-X}\right)^{n-3 / 2}$ is $\searrow$ in $0<x<1$, from $\infty$ to 1 .

We computed the values of $\Phi(x, n)$ for $0<x<1$ and $2 \leqq n<(11+\sqrt{77}) / 4$ with 
step $1 / 100$ by a personal computor, fully taking account of Propositions $3,4,5$, Lemmas 6.1, 6.2 and the above Remark, and obtained the inequality

$$
\Phi(x, n)>0
$$

for these values of $x$ and $n$, from which we can have the same inequality for all pairs of $x$ and $n$ of the above intervals of $x$ and $n$, thus we have proved the following

Proposition 6. We have

$$
\Psi(x, n)>0 \quad \text { for } 0<x<1 \text { and } 2 \leqq n \leqq \frac{11+\sqrt{ } 77}{4} .
$$

By means of the arguments developed in $\S 2$ and $\S 3$, we have proved the following

Proposition 7. Regarding $\rho\left(x, x_{1}\right)$ defined by (2.9) we have

$$
\rho\left(x, x_{1}\right)>0 \quad \text { for } x_{0}=X_{n}^{-1}\left(x_{1}\right) \leqq x<1 \text { with } 2 \leqq n \leqq \frac{11+\sqrt{77}}{4} .
$$

Thus, we have proved Main Theorem, combining the results in (IV)-(IX).

MAIN THEOREM. The period $T$ as a function of $\tau=\left(x_{1}-1\right) /(n-1)$ and $n$ is monotone decreasing with respect to $n(\geqq 2)$ for any fixed $\tau(0<\tau<1)$.

\section{REFERENCES}

[1] S.S. Chern, M. do Carmo and S. Kobayashi, Minimal Submanifolds of a sphere with second fundamental form of constant length, Functional Analysis and Related Fields, Springer-Verlag, 1970, 60-75.

[2] S. FuruYA, On periods of periodic solutions of a certain nonlinear differential equation, Japan-United States Seminar on Ordinary Differential and Functional Equations, Lecture Notes in Mathematics, Springer-Verlag, 243 (1971), 320-323.

[3] W. Y. HSIANG AND H.B. LAwson, JR., Minimal submanifolds of low cohomogeneity, J. Diff. Geometry, 5 (1970), 1-38.

[4] T. OTsuki, Minimal hypersurfaces in a Riemannian manifold of constant curvature, Amer. J. Math., 92 (1970), 145-173.

[5] T. OTsukI, On integral inequalitres related with a certain nonlinear differential equation, Proc. Japan Acad., 48 (1972), 9-12.

[6] T. Otsuki, On a 2-dimensional Riemannian manifold, Differential Geometry, in honor of K. Yano, Kinokuniya, Tokyo, 1972, 401-414.

[7] T. Otsuki, On a family of Riemannian manifolds defined on an $m$-disk, Math. J. Okayama Univ., 16 (1973), 85-97.

[8] T. OTSUKI, On a bound for periods of solutions of a certain nonlinear differential equation (I), J. Math. Soc. Japan, 26 (1974), 206-233. 
[9] T. Отsuki, On a bound for periods of solutions of a certain nonlinear differential equation (II), Funkcialaj Ekvacioj. 17 (1974), 193-205.

[10] T. OTsuki, Geodesics of $O_{n}^{2}$ and an analysis on a related Riemann surface, Tôhoku Math. J., 28 (1976), 411-427.

[11] T. OTsuki, A certain property of geodesics of the family of Riemannian manifolds $O_{n}^{2}$ (I), Proc. of Japan-United States Seminar on Minimal Submanifolds, including Geodesics, Kaigai, Tokyo, 1978, 173-192.

[12] T. OTsuki, A certain property of geodesics of the family of Riemannian manifolds $O_{n}^{2}$ (II), Kodai Math. J., 2 (1979), 211-242.

[13] T. Otsuki, A certain property of geodesics of the family of Riemannian manifolds $O_{n}^{2}$ (III), Kodai Math. J., 4 (1981), 28-70.

[14] $\mathrm{T} \cdot$ OTsuki, A certain property of geodesics of the family of Riemannian manifolds $O_{n}^{2}$ (IV), Kodai Math. J., 5 (1982), 160-199.

[15] T. OTsukI, A certain property of geodesics of the family of Riemannian manifolds $O_{n}^{2}(\mathrm{~V})$, Kodai Math. J., 5 (1982), 454-481.

[16] T. OTsuki, A certain property of geodesics of the family of Riemannian manifolds $O_{n}^{2}$ (VI), Kodai Math. J., 7 (1984), 326-364.

[17] T. OTsukı, Certain inequalities related with a non-linear differential equation (I), (II), TRU Math., 20 (1984), 23-68, 69-89.

[18] T. OTsuki, A certain property of geodesics of the family of Riemannian manifolds $O_{n}^{2}$ (VII), Kodai Math. J., 8 (1985), 375-419.

[19] T. OTsukı, Certain inequalities and constants related with a non-linear differential equation, TRU Math., 21 (1985), 5-35.

[20] T. OTsuki, A certain property of geodesics of the family of Riemannian manifolds $O_{n}^{2}$ (VIII), Kodai Math. J., 9 (1986), 272-307.

[21] T. OTsukI, Certain inequalities and constants related with a non-linear dfferential equation (II), TRU Math., 21 (1985), 241-268.

[22] T. OTsukI, A certain property of geodesics of the family of Riemannian manifolds $O_{n}^{2}$ (IX), Kodai Math. J., 11 (1988), 86-128.

[23] T. OTsukI, Certain inequalities and constants related with a non-linear differential equation (III), TRU Math., 23 (1987), 197-240.

[24] T. OTsukI, Certain inequatities related with a non-linear differential equation (III), TRU Math., 24 (1988), 103-134.

[25] T. Otsuki, A family of 2-dimensional Riemannian manifolds joining the noneuclidean planes, TRU Math., 17 (1981), 129-139.

[26] M. MAEDA AND T. Otsuki, Models of the Riemannian manifolds $O_{n}^{2}$ in the Lorentzian 4-space, J. Diff. Geometry, 9 (1974), 97-108.

[27] M. URABE, Computations of periods of a certain nonlinear autonomous Oscilations, Study of argorithms of numerical computations, Sūrikaiseki Kenkyūsho Kōkyu-roku, 149 (1972), 111-129 (Japanese).

Department of Mathematics

SCIENCE University of TOKYO

W AKAMIYA-CHO 26, SHINJUKU-KL

TOKYO, JAPAN 162 